\title{
ON THE INTERACTION BETWEEN IMIDAZOLES AND ZINC SALTS. THE ROLE OF COUNTERIONS AND OF SUBSTITUENTS
}

\author{
M. Pellei, C. Pettinari*, A. Cingolani, and A. Lacchè \\ Dipartimento di Scienze Chimiche, Università degli Studi, via S. Agostino I, 1-62032 Camerino, MC, Italy
}

\begin{abstract}
The reactions between benzimidazole $\left(\mathrm{L}^{1}\right)$, 4-phenylmidazole $\left(\mathrm{L}^{2}\right), 4$-methylimidazole $\left(\mathrm{L}^{3}\right), 2$ phenylimidazole $\left(\mathrm{L}^{4}\right)$ and $\mathrm{ZnX} 2\left(\mathrm{X}=\mathrm{Cl}, \mathrm{Br}, \mathrm{I}, \mathrm{NO}_{3}, \mathrm{ClO}_{4}, \mathrm{CH}_{3} \mathrm{COO}, \mathrm{CF}_{3} \mathrm{COO}, \mathrm{CF}_{3} \mathrm{SO}_{3}\right.$ or $\left.\mathrm{BF}_{4}\right)$ have been investigated. 31 novel adducts were obtained and characterized by analytical (elemental analysis and conductivity measurements) and spectral (IR, far IR and ${ }^{1} H$ NMR) data. The ligands $L^{1}, L^{2}, L^{3}$ and $L^{4}$ behave always in the monodentate $\mathrm{N}$-donor fashion yielding $2: 1\left(\mathrm{~L}_{2} \mathrm{ZnX}, \mathrm{L}=\mathrm{L}^{1}\right.$ and $\left.\mathrm{L}^{4}\right), 3: 1\left(\mathrm{~L}_{3} \mathrm{ZnX}, \mathrm{L}_{2}=\mathrm{L}^{2}, \mathrm{~L}^{\prime}\right.$ and $\left.L^{4}\right), 4: 1\left(L_{4} Z_{n X}, L=L^{1}, L^{2}, L^{3}\right.$ and $\left.L^{4}\right), 5: 1\left(\left[L_{s} Z n\right] X_{2}, L=L^{1}\right)$ and $6: 1$ derivatives $\left(\left[L_{6} Z n\right] X_{2}, L=L^{2}\right)$. The behavior of the adducts in acetone and chlorinated solvents is also discussed. Comparison has been made with related complexes of imidazoles.
\end{abstract}

\section{Introduction}

Zinc is recognized to be essential to all forms of life, ${ }^{1.4}$ and a large number of diseases and congenital disorders have been traced to zinc deficiency. In 1940 carbonic anhydrase was shown to be a zinc enzyme, and in 1955 carboxypeptidase became the second recognized zinc enzyme. Since then more than 300 other zinc enzymes have been reported, and about 20 have known structures based on X-ray study in the crystalline state and/or NMR data for solutions, ${ }^{s}$ most of them being imidazole derivatives. In addition there are a number of cases in which $\mathrm{Zn}^{+2}$ ions are present to provide structural rigidity to a certain region of the protein. ${ }^{6}$ The most notable of these are the "zinc fingers" and some related "twists" and "clusters". These occur in DNA binding proteins where they stabilize the correct binding sites. Finally there are the metallothionines, which store zinc. ${ }^{7.8}$

On the other hand imidazole is a molecule of considerable interest as versatile donor and potential fungicide ${ }^{9}$ and also of great biochemical importance ${ }^{10-11}$ : this five-membered nitrogen heterocycle in fact occurs in proteins as part of the side chain of the amino acid histidine, ${ }^{12}$ in nucleic acid structures as part of the purine moiety ${ }^{1 .}$ of adenine and guanine, and in the vitamin $B_{12}$ cohenzime as benzimidazole. ${ }^{14}$

Many studies have appeared in literature which describe the synthesis and characterization of imidazole-metal ion derivatives ${ }^{15}$ as model compounds in order to obtain indirect knowledge of the nature of the interactions involved in a proposed enzyme-metal-ion-substrate complex, but generally the simple imidazole heterocycle has been employed.

We have recently investigated the reactivity of variously substituted imidazoles with $\mathrm{Hg}(\mathrm{II}){ }^{16}$ $\left.{ }^{18} \mathrm{Au}(\mathrm{I}),{ }^{19} \mathrm{Cu}(\mathrm{I}),{ }^{20-22} \mathrm{Ag}(\mathrm{I}),{ }^{-5} \mathrm{Sn}(\mathrm{IV})\right)^{-4 \cdot-0}$ and of $\mathrm{N}$-substituted imidazoles with $\mathrm{Zn}(\mathrm{II})$ and $\mathrm{Cd}(\mathrm{II}) .^{29}$

The purpose of this paper is to extend the knowledge of the modes of action of substituted imidazoles towards zinc acceptors. Here we report the synthesis and spectroscopic characterization of new $\mathrm{Zn}(\mathrm{II})$ derivatives of benzimidazole $\left(\mathrm{L}^{\prime}\right)$, 4-phenylmidazole $\left(\mathrm{L}^{2}\right)$, 4-methylimidazole ( $\left.\mathrm{L}^{\prime}\right)$ and 2phenylimidazole $\left(\mathrm{L}^{4}\right)$ (Fig. 1). Factors affecting the stoichiometry and the coordination number are discussed and rationalized on the basis of steric and electronic arguments.

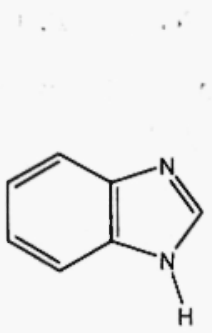

$\mathbf{L}^{\prime}$

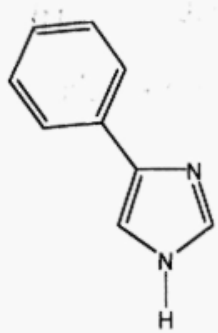

$\mathbf{L}^{2}$

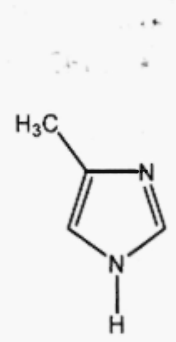

$\mathbf{L}^{3}$<smiles>c1ccc(-c2ncc[nH]2)cc1</smiles>

$\mathbf{L}^{+}$

Fig. 1. Ligands employed in this work. 


\section{Experimental Section}

The zinc(II) salts were purchased from Alfa (Karlsruhe) and Aldrich (Milwaukee) and used as received. The ligands benzimidazole $\left(\mathrm{L}^{\mathrm{l}}\right)$, 4-phenylmidazole $\left(\mathrm{L}^{2}\right)$, 4-methylimidazole $\left(\mathrm{L}^{3}\right)$ and 2 phenylimidazole $\left(\mathrm{L}^{4}\right)$ were obtained from Aldrich and were crystallized from diethyl ether/petroleum ether $(1: 2)$. The samples were dried in vacuo to constant weight $\left(20^{\circ} \mathrm{C}, \sim 0.1\right.$ Torr $)$. Elemental analyses were carried out in-house with a Fisons Instruments $1108 \mathrm{CHNSO}$-elemental analyser. IR spectra from 4000 to $150 \mathrm{~cm}^{-1}$ were recorded with a Perkin Elmer System $2000 \mathrm{FT}-\mathrm{IR}$ instrument. $\mathrm{H}$ NMR spectra $(300 \mathrm{MHz})$ were recorded on a VXR-30 9 Varian Spectrometer operating at room temperature. The chemical shifts are reported in ppm from $\mathrm{SiMe}_{4}$ ( $\mathrm{H}$ calibration by internal deuterium solvent lock).

Melting points were taken on an IA 8100 Electrothermal Instrument. The electrical resistance of solutions were measured with a Crison CDTM 522 conductimeter at room temperature.

CAUTION. The perchlorato derivatives reported in this paper may explode by shock or heating when dry. Small quantities $(<0.5 \mathrm{~g})$ of the dry products should be handled with all possible precaution. Synthesis

All experiments were carried out under a dinitrogen atmosphere. Hydrocarbon solvents were dried by distillation from sodium-potassium, dichloromethane from calcium hydride and tetrahydrofuran from $\mathrm{LiAlH}_{4}$. All solvents were outgassed with dry dinitrogen prior to use.

/(Benzimidazole $)_{2} \mathrm{ZnCl} /\left(\mathrm{H}_{2} \mathrm{O}\right)_{0.5}$ (1). A suspension of benzimidazole $(0.473 \mathrm{~g}, 4.0 \mathrm{mmol})$ in diethyl ether $(30 \mathrm{ml})$ was treated with $\mathrm{ZnCl}_{2}(0.136 \mathrm{~g}, 1.0 \mathrm{mmol})$ in ethanol $(20 \mathrm{ml})$. The reaction was stirred at room temperature for $3 \mathrm{~h}$. A colorless precipitate was formed which was filtered off and washed with diethyl ether $(2 \times 5 \mathrm{ml})\left(76 \%\right.$ yield). M.p. $228-229^{\circ} \mathrm{C}$. Found: $\mathrm{C}, 44.4 ; \mathrm{H}, 3.4 ; \mathrm{N}, 14.8$. Calc. for $\mathrm{C}_{14} \mathrm{H}_{13} \mathrm{Cl}_{2} \mathrm{~N}_{4} \mathrm{O}_{05} \mathrm{Zn}_{3} \mathrm{C}$, 44.1; H, 3.4; N, 14.7. IR (nujol, $\mathrm{cm}^{-1}$ ): $3317 \mathrm{~s} v(\mathrm{H}, \mathrm{O}), 3142 \mathrm{w} v(\mathrm{C}-\mathrm{H}), 307 \mathrm{~s}, 297 \mathrm{~s} v(\mathrm{Zn}-\mathrm{Cl}), 230 \mathrm{~m} \mathrm{v}(\mathrm{Zn}-\mathrm{N})$. ${ }^{\prime} \mathrm{H}$ NMR (Acetone): $\delta, 2.9(\mathrm{br}, 0.5 \mathrm{H}, \mathrm{H}, \mathrm{O}), 7.29-7.38(\mathrm{~m}, 4 \mathrm{H}, P h), 7.91(\mathrm{br}, 4 \mathrm{H}, P h), 8.76(\mathrm{~s}, 2 \mathrm{H}, H-2), 12.4$ (br, $2 \mathrm{H}, \mathrm{N} H) .{ }^{13} \mathrm{C}\{\mathrm{H}\}$ NMR (Acetone): $\delta, 117.0(\mathrm{br}, C-6$ and $C-9), 125.16(C-7$ and $C-8), 137.0$ (br, $C-4$ and $C-5), 144.75(C-2)$.

/(Benzimidazole) ${ }_{2} \mathrm{ZnBr}_{2} /(2)$. A suspension of benzimidazole $(0.473 \mathrm{~g}, 4.0 \mathrm{mmol})$ in diethyl ether $(30 \mathrm{ml})$ was treated with $\mathrm{ZnBr}_{2}(0.225 \mathrm{~g}, 1.0 \mathrm{mmol})$ and $\mathrm{CH}_{2} \mathrm{Cl}_{2}(20 \mathrm{ml})$. The reaction was stirred at room temperature for $3 \mathrm{~h}$. A colorless precipitate was formed which was filtered off and washed with diethyl ether $(2 \times 5 \mathrm{ml})(70 \%$ yield $)$. M.p. $243-245^{\circ} \mathrm{C}$. Found: C, 36.4; H, 2.6; N, 12.1. Calc. for $\mathrm{C}_{14} \mathrm{H}_{12} \mathrm{Br}_{2} \mathrm{~N}_{4} \mathrm{Zn}, \mathrm{C}, 36.8$; $\mathrm{H}, 2.7$; N, 12.1. IR (nujol. $\mathrm{cm}^{-1}$ ): $3107 \mathrm{wbr} v(\mathrm{C}-\mathrm{H}), 229 \mathrm{vs}$. 'H NMR (Acetone): $\delta, 7.31-7.36$ (m, $\left.4 \mathrm{H}, P h\right) 7.90$ (br, $4 \mathrm{H}, P h), 8.83(\mathrm{~s}, 2 \mathrm{H}, H-2), 12.5(\mathrm{br}, 2 \mathrm{H}, \mathrm{N} H)$.

2.2.3. I(Benzimiduzole) ${ }_{4} \mathrm{ZnI} /\left(\mathrm{H}_{2} \mathrm{O}\right)_{0.5}$ (3). A suspension of benzimidazole $(0.473 \mathrm{~g}, 4.0 \mathrm{mmol})$ in diethyl ether $(30 \mathrm{ml})$ was treated with $\mathrm{Znl}_{2}(0.319 \mathrm{~g}, 1.0 \mathrm{mmol})$ and $\mathrm{CH}_{2} \mathrm{Cl}_{2}(20 \mathrm{ml})$. The reaction was stirred at room temperature for $24 \mathrm{~h}$. The solution was then concentrated under reduced pressure; the oily residue obtained was crystallized twice from $\mathrm{CH}_{2} \mathrm{Cl}_{2}$ /diethyl ether $\left(60 \%\right.$ yield). M.p. $100-103^{\circ} \mathrm{C}$. Found: $\mathrm{C}, 42.0 ; \mathrm{H}, 3.2 ; \mathrm{N}$, 14.0. Calc. for $\mathrm{C}_{78} \mathrm{H}_{25} \mathrm{l}_{2} \mathrm{~N}_{8} \mathrm{O}_{0.5} \mathrm{Zn}, \mathrm{C}, 42.1 ; \mathrm{H}, 3.4 ; \mathrm{N}, 13.5$. IR (nujol, cm $\mathrm{cm}^{-1}$ ): $3367 \mathrm{br} v(\mathrm{H}, \mathrm{O}), 3156 \mathrm{w} v(\mathrm{C}=\mathrm{H})$, 201 vs $v(Z n-1), 227 \mathrm{~m} v(\mathrm{Zn}-\mathrm{N})$. 'H NMR (Acetone): $\delta, 4.0\left(\mathrm{br}, 1 \mathrm{H}, H_{2} \mathrm{O}\right), 7.24-7.32(\mathrm{~m}, 8 \mathrm{H}, P h), 7.75-7.77$ $(\mathrm{m}, 8 \mathrm{H}, P h), 8.57(\mathrm{~s}, 4 \mathrm{H}, \mathrm{H}-2)$.

I(Benzimidazole $)_{2} \mathrm{Zn}\left(\mathrm{NO}_{1}\right)_{2} \mathrm{~J}\left(\mathrm{H}_{2} \mathrm{O}\right)_{2}$ (4). Compound 4 was prepared similarly to compound 3, by using $\mathrm{Zn}\left(\mathrm{NO}_{i}\right)_{2}$. Recrystallized from petroleum ether $\left(78 \%\right.$ yield). M.p. $85-88^{\circ} \mathrm{C}$. Found: $\mathrm{C}, 36.4 ; \mathrm{H}, 3.5 ; \mathrm{N}, 18.2$. Calc. for $\mathrm{C}_{14} \mathrm{H}_{16} \mathrm{~N}_{6} \mathrm{O}_{8} \mathrm{Zn}, \mathrm{C}, 37.2 ; \mathrm{H}, 3.5 ; \mathrm{N}, 18.1$. IR (nujol, $\left.\mathrm{cm}^{-1}\right)$ : $3322 \mathrm{wbr} v(\mathrm{H}, \mathrm{O}), 3168 \mathrm{w} v(\mathrm{C}-\mathrm{H})$, $1750 \mathrm{vw}$, $1734 v w v_{1}+v_{4}\left(\mathrm{NO}_{3}\right), 1377 v s \mathrm{v}\left(\mathrm{NO}_{3}\right), 287 \mathrm{~m}, 273 \mathrm{w} v(\mathrm{Zn}-\mathrm{O}), 232 \mathrm{~m}, 205 \mathrm{~m} v(\mathrm{Zn}-\mathrm{N}){ }^{1} \mathrm{H}$ NMR (Acetone): $\delta$, $3.70\left(\mathrm{br}, 4 \mathrm{H}, \mathrm{H}_{2} \mathrm{O}\right), 7.43-7.51(\mathrm{~m}, 4 \mathrm{H}, \mathrm{Ph}), 7.76-7.81(\mathrm{~m}, 4 \mathrm{H}, P h), 8.78(\mathrm{~s}, 2 \mathrm{H}, \mathrm{H}-2), 12.8(\mathrm{br}, 2 \mathrm{H}, \mathrm{NH})$. Cond. (Acetone, conc. $1.02 \times 10^{-9} \mathrm{M}$ ): $\Lambda_{\mathrm{m}} 7.5 \Omega^{-1} \mathrm{~cm}^{-} \mathrm{mol}^{-1}$.

/(Benzimiduzole $\left.)_{4} \mathrm{Zn}(\mathrm{ClO})_{4}\right)_{2}\left(\mathrm{H}_{2} \mathrm{O}\right)(5)$. Compound 5 was prepared similarly to compound 2 , by using $\mathrm{Zn}\left(\mathrm{ClO}_{4}\right)_{7}\left(92 \%\right.$ yield). M.p. $162-165^{\circ} \mathrm{C}$. Found: $\mathrm{C}, 44.6 ; \mathrm{H}, 3.5 ; \mathrm{N}, 14.8$. Calc. for $\mathrm{C},{ }_{8} \mathrm{H},{ }_{6} \mathrm{Cl}_{3} \mathrm{~N}_{8} \mathrm{O}_{9} \mathrm{Zn}, \mathrm{C}$, $44.7 ; \mathrm{H}, 3.6 ; \mathrm{N}, 14.8$. IR (nujol. $\left.\mathrm{cm}^{-1}\right): 3301 \mathrm{mbr} v(\mathrm{H}, \mathrm{O}), 3114 \mathrm{w} v(\mathrm{C}-\mathrm{H}), 1155 \mathrm{w}, 1094 \mathrm{vsbr}, 1008 \mathrm{w}, 979 \mathrm{w}$ $\mathrm{v}\left(\mathrm{ClO}_{4}\right), 294 \mathrm{~m}, 229 \mathrm{~m} v(\mathrm{Zn}-\mathrm{O})$. ${ }^{\mathrm{i}} \mathrm{H}$ NMR (Acetone): $\delta, 2.22(\mathrm{~s}, 2 \mathrm{H}, H, \mathrm{O}), 7.35-7.39(\mathrm{~m}, 8 \mathrm{H}, P h), 7.72-7.76$ $(\mathrm{m}, 8 \mathrm{H}, P h), 9.03(\mathrm{~s}, 4 \mathrm{H}, H-2), 12.8(\mathrm{br}, 4 \mathrm{H}, \mathrm{N} H) .{ }^{13} \mathrm{C}\left\{{ }^{\prime} \mathrm{H}\right\}$ NMR (Acetone): $\delta, 115.97(C-6$ and $C-9), 125.65$ (C-7 and $C-8), 134.5,136.0($ br, $C-4$ and $C-5), 145.10(C-2)$.

/(Benzimidazole $)_{2} \mathrm{Zn}\left(\mathrm{CH}_{3} \mathrm{COO}\right)_{2} /$ (6). Compound 6 was prepared similarly to compound $\mathbf{I}$, by using $\mathrm{Zn}\left(\mathrm{CH}_{3} \mathrm{COO}\right)$, and washed with ethanol/diethyl ether $(67 \%$ yield $)$. Insoluble in all common organic solvents. M.p. $320-323^{\circ} \mathrm{C}$. Found: $\mathrm{C}, 51.5 ; \mathrm{H}, 4.3 ; \mathrm{N}, 13.4$. Calc. for $\mathrm{C}_{18} \mathrm{H}_{18} \mathrm{Cl}_{2} \mathrm{~N}_{4} \mathrm{O}_{4} \mathrm{Zn}, \mathrm{C}, 49.6 ; \mathrm{H}, 3.6 ; \mathrm{N}, 15.2$. IR (nujol, $\mathrm{cm}^{-1}$ ): $3159 \mathrm{wbr}, 308 \mathrm{lw} v(\mathrm{C}-\mathrm{H}), 1610 \mathrm{~s}, 1377 \mathrm{vsbr} v(\mathrm{COO}), 285 \mathrm{~s} v(\mathrm{Zn}-\mathrm{O})$.

2.2.7. /(Benzimidazole), $\mathrm{Zn}\left(\mathrm{CF}_{3} \mathrm{SO}_{3}\right)_{2} /(7)$. Compound 7 was prepared similarly to compound 2 , by using $\mathrm{Zn}\left(\mathrm{CF}_{3} \mathrm{SO}_{3}\right)_{2}(21 \%$ yield $)$. M.p. $128-131^{\circ} \mathrm{C}$. Found: $\mathrm{C}, 43.1 ; \mathrm{H}, 2.9 ; \mathrm{N}, 13.4$. Calc. for $\mathrm{C}_{30} \mathrm{H}_{24} \mathrm{~F}_{6} \mathrm{~N}_{8} \mathrm{O}_{6} \mathrm{~S}_{2} \mathrm{Zn}_{2} \mathrm{C}$, 43.0; H, 2.8: N, 13.4. IR (nujol, $\mathrm{cm}^{-1}$ ): $3143 w b r v(\mathrm{C}-\mathrm{H}), 1303 \mathrm{mbr}, 640 \mathrm{~s}, 516 \mathrm{~m} v\left(\mathrm{SO}_{3}\right), 1168 \mathrm{~m} v(\mathrm{C}-\mathrm{F})$. ${ }^{1} \mathrm{H}$ NMR (Acetone): $\delta, 7.39-7.44(\mathrm{~m}, 8 \mathrm{H}, P h), 7.75-7.80(\mathrm{~m}, 8 \mathrm{H}, P h), 9.10(\mathrm{~s}, 4 \mathrm{H}, H-2)$.

/(Benzimidazole) $Z n\left(C_{3} \boldsymbol{S O}_{3}\right)_{2} /(8)$. Benzimidazole $(0.709 \mathrm{~g}, 6.0 \mathrm{mmol})$ was added to an ethanol solution $(30 \mathrm{ml})$ of $\mathrm{Zn}\left(\mathrm{CF}_{3} \mathrm{SO}_{3}\right)_{2}(0.363 \mathrm{~g}, 1.0 \mathrm{mmol})$. The reaction was stirred at room temperature for $3 \mathrm{~h}$. A colorless precipitate was formed, which was filtered off and washed with ethanol $(3 \times 5 \mathrm{ml})$ and recrystallized from $\mathrm{CH}_{2} \mathrm{Cl}_{2}$ /petroleum ether $\left(60 \%\right.$ yield). M.p. $97-100^{\circ} \mathrm{C}$. Found: $\mathrm{C}, 46.6 ; \mathrm{H}, 3.2 ; \mathrm{N}, 14.7$. Calc. for $\mathrm{C}_{37} \mathrm{H}_{30} \mathrm{~F}_{6} \mathrm{~N}_{8} \mathrm{O}_{6} \mathrm{~S} 2 \mathrm{Zn}, \mathrm{C}, 47.6 ; \mathrm{H}, 3.3 ; \mathrm{N}, 14.8$. IR (nujol, $\mathrm{cm}^{-1}$ ): $3146 \mathrm{wbr} v(\mathrm{C}-\mathrm{H}), 1272 \mathrm{vsbr}, 638 \mathrm{~s}$, $515 \mathrm{~m} v\left(\mathrm{SO}_{3}\right)$. $1168 \mathrm{~s} \vee(\mathrm{C}-\mathrm{F})$. 'H NMR (Acetone): $\delta .7 .29-7.33(\mathrm{~m}, 10 \mathrm{H}, P h), 7.60-7.80(\mathrm{~m}, 10 \mathrm{H}, P h), 8.79(\mathrm{~s}$, $5 \mathrm{H}, H-2), 9.2(\mathrm{br}, 5 \mathrm{H}, \mathrm{N} H)$. 


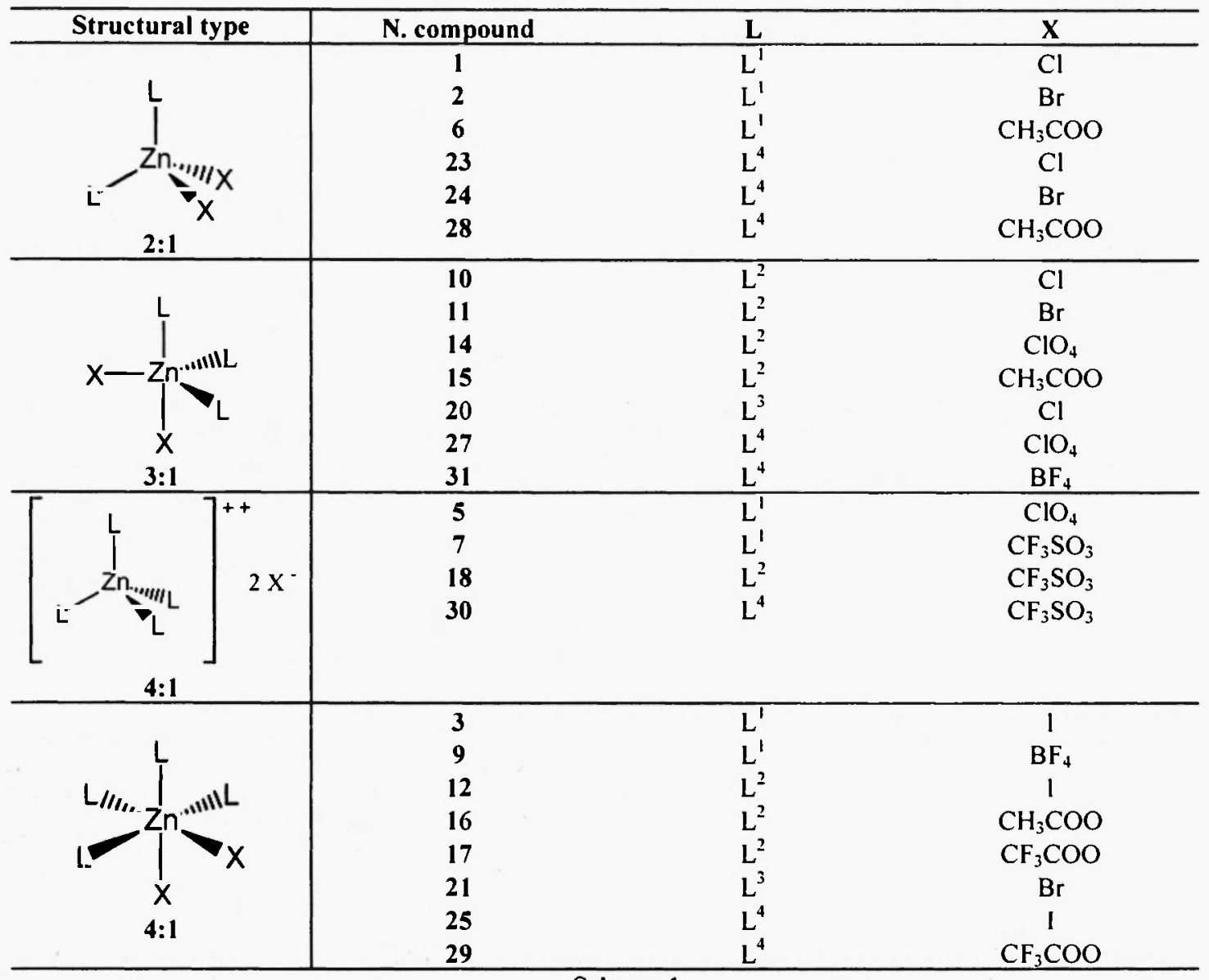

Scheme 1

/(Benzimidazole) $)_{4} Z n\left(B F_{4}\right)_{2} /(9)$. Benzimidazole $(0.473 \mathrm{~g}, 4.0 \mathrm{mmol})$ was added to an ethanol solution (30 $\mathrm{ml})$ of $\mathrm{Zn}\left(\mathrm{BF}_{4}\right),(0.239 \mathrm{~g}, 1.0 \mathrm{mmol})$. The reaction was stirred at room temperature for $3 \mathrm{~h}$. A colorless precipitate was formed, which was filtered off and washed with ethanol $(2 \times 5 \mathrm{ml})$ and recrystallized from $n$ pentane $\left(28 \%\right.$ yield). M.p. $13 \mathrm{I}-134^{\circ} \mathrm{C}$. Found: $\mathrm{C}, 47.3 ; \mathrm{H}, 3.4 ; \mathrm{N}, 15.8$. Calc. for $\mathrm{C}_{8} \mathrm{H}_{24} \mathrm{~B} \mathrm{~F}_{8} \mathrm{~N}_{8} \mathrm{Zn}, \mathrm{C}, 51.0$; $\mathrm{H}, 3.9 ; \mathrm{N}, 17.0$. IR (nujol, $\mathrm{cm}^{-1}$ ): $3150 \mathrm{wbr} v(\mathrm{C}-\mathrm{H}), 1154 \mathrm{vs}, 1078 \mathrm{mbr}, 102 \mathrm{Imbr}, 768 \mathrm{w}, 520 \mathrm{w} \mathrm{v}\left(\mathrm{BF}_{4}\right), 200 \mathrm{~s}$ $\mathrm{v}(\mathrm{Zn}-\mathrm{N}), 280 \mathrm{~m}, 268 \mathrm{vw}, 247 \mathrm{w}, 225 \mathrm{w} v(\mathrm{Zn}-\mathrm{F})$. 'H NMR (Acetone): $\delta, 7.28-7.34(\mathrm{~m}, 8 \mathrm{H}, P h), 7.60-7.80(\mathrm{~m}$, $8 \mathrm{H}, P h), 8.4(\mathrm{br}, 4 \mathrm{H}, \mathrm{N} H), 8.75(\mathrm{~s}, 4 \mathrm{H}, H-2)$.

/(4-phenylimidazole) $)_{3} \mathrm{ZnCl}_{2} /\left(\mathrm{H}_{2} \mathrm{O}\right)$ (10). An ethanol solution $(20 \mathrm{ml})$ of $\mathrm{ZnCl}_{2}(0.136 \mathrm{~g}, 1.0 \mathrm{mmol})$ was added to a diethyl ether suspension $(30 \mathrm{ml})$ of 4-phenylimidazole $(0.579 \mathrm{~g}, 4.0 \mathrm{mmol})$. The reaction was stirred at room temperature for $24 \mathrm{~h}$. The solution was then concentrated under reduced pressure; the colorless precipitate obtained was recrystallized twice from $\mathrm{CH}_{2} \mathrm{Cl}_{2} /$ diethyl ether $(92 \%$ yield). M.p. 106 $108^{\circ} \mathrm{C}$. Found: $\mathrm{C}, 55.3 ; \mathrm{H}, 4.5 ; \mathrm{N}, 14.3$. Calc. for $\mathrm{C}_{27} \mathrm{H}_{26} \mathrm{Cl}_{2} \mathrm{~N}_{6} \mathrm{OZn}, \mathrm{C}, 55.7 ; \mathrm{H}, 4.7 ; \mathrm{N}, 13.9$. IR (nujol, $\mathrm{cm}^{-1}$ ): $3123 \mathrm{br} v(\mathrm{C}-\mathrm{H}), 303 \mathrm{~s}, 280 \mathrm{~m} v(\mathrm{Zn}-\mathrm{Cl}), 224 \mathrm{w} v(\mathrm{Zn}-\mathrm{N})$. H NMR (Acetone): $\delta, 2.9$ (br, $2 \mathrm{H}, \mathrm{H}_{2} \mathrm{O}$ ), 7.20-7.50, $\left(\mathrm{m}, 9 \mathrm{H}, \mathrm{C}_{6} H_{5}\right), 7.56(\mathrm{~s}, 3 \mathrm{H}, H-5), 7.78\left(\mathrm{~d}, 6 \mathrm{H}, \mathrm{C}_{6} H_{5}\right), 8.07(\mathrm{~s}, 3 \mathrm{H}, H-2), 12.2(\mathrm{br}, 3 \mathrm{H}, \mathrm{N} H) .{ }^{13} \mathrm{C}\left\{{ }^{1} \mathrm{H}\right\} \mathrm{NMR}$ (Acetone): $\delta, 120.53(\mathrm{br}, C-5), 125.83(C-7$ and $C-11), 128.33(C-9), 129.65(C-8$ and $C-10), 132.0,136.0$ (br, $C-5$ and $C-6$ ), $138.11(C-2)$. Cond. $\left(\mathrm{CH}, \mathrm{Cl}\right.$, conc. $\left.1.04 \times 10^{-3} \mathrm{M}\right):$ ): $\Lambda_{\mathrm{m}} 0.07 \Omega^{-1} \mathrm{~cm}^{2} \mathrm{~mol}^{-1}$; (Acetone, conc. $\left.1.09 \times 10^{-5} \mathrm{M}\right):$ ): $\Lambda_{\mathrm{m}} 2.6 \Omega^{-1} \mathrm{~cm}^{2} \mathrm{~mol}^{-1}$.

/(4-phenylimidazole), $\mathrm{ZnBr}_{2} /(11)$. A diethyl ether solution $(20 \mathrm{ml})$ of $\mathrm{ZnBr},(0.225 \mathrm{~g}, 1.0 \mathrm{mmol})$ was added to a dichloromethane solution $(30 \mathrm{ml})$ of 4 -phenylimidazole $(0.579 \mathrm{~g}, 4.0 \mathrm{mmol})$. The reaction was stirred at room temperature for $24 \mathrm{~h}$. The solution was then concentrated under reduced pressure; the colorless precipitate obtained was recrystallized from diethyl ether $\left(76 \%\right.$ yield). M.p. $103-107^{\circ} \mathrm{C}$. Found: $\mathrm{C}, 49.3 ; \mathrm{H}$, 3.7; N, 12.8. Calc. for $\mathrm{C}_{27} \mathrm{H}_{24} \mathrm{Br}_{3} \mathrm{~N}_{6} \mathrm{Zn}, \mathrm{C}, 50.9 ; \mathrm{H}, 4.2 ; \mathrm{N}$, 12.9. IR (nujol, $\mathrm{cm}^{-1}$ ): $3129 \mathrm{br} v(\mathrm{C}-\mathrm{H}), 304 \mathrm{w}, 226 \mathrm{~m}$ $v(\mathrm{Zn}-\mathrm{Br}), 23 \mathrm{Im} v(\mathrm{Zn}-\mathrm{N})$. 'H NMR (Acetone): $\delta, 7.20-7.45\left(\mathrm{~m}, 9 \mathrm{H}, \mathrm{C}_{6} H_{5}\right), 7.59(\mathrm{~s}, 3 \mathrm{H}, H-5), 7.79(\mathrm{~d}, 6 \mathrm{H}$, $\left.\mathrm{C}_{6} H_{5}\right), 8.10(\mathrm{br}, 3 \mathrm{H}, H-2) .{ }^{13} \mathrm{C}\left\{{ }^{1} \mathrm{H}\right\}$ NMR (Acetone): $\delta, 119.93(\mathrm{br}, \mathrm{C}-5), 125.74(\mathrm{C}-7$ and $\mathrm{C}-1 \mathrm{l}), 128.13$ (C9), $129.59(C-8$ and $C-10), 131.8,136.0$ (br, $C-5$ and $C-6), 137.87(C-2)$. Cond. $\left(\mathrm{CH}_{2} \mathrm{Cl}_{2}\right.$, conc. $\left.1.03 \times 10^{-5} \mathrm{M}\right)$ : ): $\Lambda_{\mathrm{m}} 0.1 \Omega^{-1} \mathrm{~cm}^{2} \mathrm{~mol}^{-1}$; (Acetone, conc. $1.02 \times 10^{-3} \mathrm{M}$ ): $): \Lambda_{\mathrm{m}} 1.9 \Omega^{-1} \mathrm{~cm}^{-2} \mathrm{~mol}^{-1}$.

(14-phenylimidazole), $\mathrm{ZnI}_{2} J\left(\mathrm{H}_{2} \mathrm{O}\right)_{15}(12)$. A diethyl ether solution $(20 \mathrm{ml})$ of $\mathrm{Znl} 2(0.319 \mathrm{~g}, 1.0 \mathrm{mmol})$ was added to a dichloromethane solution $(30 \mathrm{ml})$ of 4-phenylimidazole $(0.579 \mathrm{~g}, 4.0 \mathrm{mmol})$. The reaction was 
stirred at room temperature for $24 \mathrm{~h}$. The solution was then concentrated under reduced pressure; the oily residue obtained was recrystallized from diethyl ether $\left(47 \%\right.$ yield). M.p. $96-102^{\circ} \mathrm{C}$. Found: $\mathrm{C}, 47.8 ; \mathrm{H}, 3.7$; $\mathrm{N}, 12.4$. Calc. for $\mathrm{C}_{36} \mathrm{H}_{3} \mathrm{l}, \mathrm{N}_{8} \mathrm{O}_{0.5} \mathrm{Zn}, \mathrm{C}, 48.3 ; \mathrm{H}, 3.9 ; \mathrm{N}, 12.4$. IR (nujol, $\mathrm{cm}^{-1}$ ): $3398 w b r v(\mathrm{H}, \mathrm{O}), 3124 \mathrm{w} v(\mathrm{C}-$ $\mathrm{H}), 20 \mathrm{l}$ vs $v(\mathrm{Zn}-\mathrm{l}), 223 \mathrm{~m} v(\mathrm{Zn}-\mathrm{N})$. ${ }^{1} \mathrm{H}$ NMR (Acetone): $\delta, 3.0(\mathrm{br}, 1 \mathrm{H}, H, \mathrm{O}), 7.20-7.50\left(\mathrm{~m}, 12 \mathrm{H}, \mathrm{C}_{6} H_{5}\right), 7.62$ (s, $4 \mathrm{H}, \mathrm{H}-5), 7.79\left(\mathrm{~d}, 8 \mathrm{H}, \mathrm{C}_{6} H_{5}\right), 8.04(\mathrm{br}, 4 \mathrm{H}, \mathrm{H}-2)$. Cond $\left(\mathrm{CH}_{2} \mathrm{Cl}\right.$, conc. $\left.1.01 \times 10^{-3} \mathrm{M}\right): \Lambda_{\mathrm{m}} 0.9 \Omega^{\circ}$ ${ }^{\prime} \mathrm{cm}^{2} \mathrm{~mol}^{-1}$; (Acetone, conc. $\left.0.99 \times 10^{-3} \mathrm{M}\right):$ ): $\Lambda_{\mathrm{In}} 8.1 \Omega^{-1} \mathrm{~cm}^{-2} \mathrm{~mol}^{-1}$.

/(4-phenylimilazole ${ }_{3} \mathrm{Zn}\left(\mathrm{NO}_{3}\right)_{2} /\left(\mathrm{H}_{2} \mathrm{O}\right)_{1 . j}(13)$. A diethyl ether suspension $(20 \mathrm{ml})$ of $\mathrm{Zn}\left(\mathrm{NO}_{3}\right)_{2}(0.189 \mathrm{~g}, 1.0$ mmol) was added to a dichloromethane solution $(30 \mathrm{ml})$ of 4 -phenylimidazole $(0.579 \mathrm{~g}, 4.0 \mathrm{mmol})$. The reaction was stirred at room temperature for $24 \mathrm{~h}$. A colorless precipitate was formed, which was filtered off and washed with diethyl ether $(2 \times 5 \mathrm{ml})(72 \%$ yield $)$. M.p. 157-161 ${ }^{\circ} \mathrm{C}$. Found: $\mathrm{C}, 51.2 ; \mathrm{H}, 4.0 ; \mathrm{N}, 17.7 . \mathrm{Calc}$. for $\mathrm{C}_{27} \mathrm{H}_{25} \mathrm{~N}_{8} \mathrm{O}_{6} ; \mathrm{Zn}, \mathrm{C}, 51.4 ; \mathrm{H}, 4.0 ; \mathrm{N}, 17.8$. IR (nujol, $\mathrm{cm}^{-1}$ ): 3542w, 3486w v( $\left.\mathrm{H}_{2} \mathrm{O}\right), 3145 w b r, 3121 \mathrm{w} v(\mathrm{C}-$ H). $1753 v w v_{1}+v_{4}\left(\mathrm{NO}_{3}\right), 1377 v s v\left(\mathrm{NO}_{3}\right), 304 \mathrm{vw}, 273 \mathrm{vw} v(\mathrm{Zn}-\mathrm{O}), 215 \mathrm{w} v(\mathrm{Zn}-\mathrm{N})$. ' H NMR (Acetone): $\delta, 2.7$ (br, $1 \mathrm{H}, H, \mathrm{O}), 7.37-7.51,\left(\mathrm{~m}, 9 \mathrm{H}, \mathrm{C}_{6} \mathrm{H}_{5}\right), 7.71 .(\mathrm{s}, 3 \mathrm{H}, H-5), 7.79\left(\mathrm{~d}, 6 \mathrm{H}, \mathrm{C}_{6} H_{5}\right), 8.37$ (s, 3H, $\left.H-2\right)$. Cond. (Acetone, conc. $\left.1.01 \times 10^{-5} \mathrm{M}\right):$ ): $\Lambda_{\mathrm{m}} 16.2 \Omega^{-1} \mathrm{~cm}^{-} \mathrm{mol}^{-1}$.

/(4-phenylimidazole $)_{2} \mathrm{Zn}(\mathrm{ClO})_{2} /\left(\mathrm{H}_{2} \mathrm{O}\right)(14)$. Compound 14 was prepared similarly to compound 12, by using $\mathrm{Zn}\left(\mathrm{ClO}_{4}\right)_{2}$. Recrystallized from $\mathrm{CH}, \mathrm{Cl}_{2} /$ diethyl ether $\left(60 \%\right.$ yield). M.p. $107-110^{\circ} \mathrm{C}$. Found: $\mathrm{C}, 45.4 ; \mathrm{H}$, 3.7; N, 11.8. Calc. for $\mathrm{C}_{77} \mathrm{H}_{2} \mathrm{Cl}_{2} \mathrm{~N}_{6} \mathrm{O}_{9} \mathrm{Zn}, \mathrm{C}, 45.8 ; \mathrm{H}, 4.1 ; \mathrm{N}, 11.8$. IR (nujol, $\mathrm{cm}^{-1}$ ): $3317 \mathrm{wbr} v(\mathrm{H}, \mathrm{O}), 3145 w$ $v(\mathrm{C}-\mathrm{H}), 1150 \mathrm{wbr}, 1075 \mathrm{mbr}, 969 \mathrm{w}, 644 \mathrm{~m}, 617 \mathrm{vs} v\left(\mathrm{NO}_{3}\right), 226 \mathrm{w} v(\mathrm{Zn}-\mathrm{O})$. 'H NMR (Acetone): $\delta, 2.9$ (br, $2 \mathrm{H}$, $\left.H_{2} \mathrm{O}\right), 7.30-7.52\left(\mathrm{~m}, 9 \mathrm{H}, \mathrm{C}_{6} H_{5}\right), 7.76(\mathrm{~s}, 3 \mathrm{H}, H-5), 7.80\left(\mathrm{~d}, 6 \mathrm{H}, \mathrm{C}_{6} H_{5}\right), 8.53(\mathrm{~s}, 3 \mathrm{H}, H-2), 11.5(\mathrm{br}, 3 \mathrm{H}, \mathrm{N} H)$. /(4-phenylimidazole $)_{3} \mathrm{Zn}\left(\mathrm{CH}_{3} \mathrm{COO}\right)_{2}$ / (15). A diethyl ether suspension $(20 \mathrm{ml})$ of $\mathrm{Zn}\left(\mathrm{CH}{ }_{3} \mathrm{COO}\right)_{2}(0.183 \mathrm{~g}$, $1.0 \mathrm{mmol})$ was added to a dichloromethane solution $(30 \mathrm{ml})$ of 4 -phenylimidazole $(0.579 \mathrm{~g} .4 .0 \mathrm{mmol})$. The reaction was stirred at room temperature for $24 \mathrm{~h}$. The solution was then concentrated under reduced pressure; the oily residue obtained was recrystallized from $\mathrm{CHCl}_{3}\left(60 \%\right.$ yield). M.p. $231-234^{\circ} \mathrm{C}$. Found: $\mathrm{C}$, 59.9; H, 4.9; N, 13.8. Calc. for $\mathrm{C}_{31} \mathrm{H}_{30} \mathrm{~N}_{6} \mathrm{O}_{4} \mathrm{Zn}, \mathrm{C}, 60.4 ; \mathrm{H}, 4.9 ; \mathrm{N}, 13.6$. IR (nujol, $\mathrm{cm}^{-1}$ ): $3126 \mathrm{wbr} v(\mathrm{C}-\mathrm{H}$ ), $1582 \mathrm{sbr}, 1376 \mathrm{vs} \vee(\mathrm{COO}), 27 \mathrm{lmbr} v(\mathrm{Zn}-\mathrm{O}), 20 \mathrm{lw} v(\mathrm{~N}-\mathrm{N}) .{ }^{1} \mathrm{H}$ NMR (Acetone): $\delta, 1.95\left(\mathrm{~s}, 6 \mathrm{H}, \mathrm{CH} \mathrm{COO}_{3}\right.$ ), 7.10- $7.40\left(\mathrm{~m}, 9 \mathrm{H}, \mathrm{C}_{6} H_{5}\right), 7.50-7.90\left(\mathrm{~m}, 6 \mathrm{H}, \mathrm{C}_{6} H_{5}\right), 7.55(\mathrm{br}, 3 \mathrm{H}, H-5), 7.70(\mathrm{br}, 3 \mathrm{H}, H-2)$.

/(4-phenylimidazole), $\mathrm{Zn}\left(\mathrm{CH}_{3} \mathrm{COO}\right), \gamma\left(\mathrm{H}_{2} \mathrm{O}\right)$ (16). A dichloromethane suspension $(20 \mathrm{ml})$ of $\mathrm{Zn}\left(\mathrm{CH} \quad{ }_{3} \mathrm{COO}\right)_{2}$ $(0.183 \mathrm{~g}$. $1.0 \mathrm{mmol})$ was added to an ethanol solution $(30 \mathrm{ml})$ of 4 -phenylimidazole $(0.579 \mathrm{~g}, 4.0 \mathrm{mmol})$. The reaction was stirred at room temperature for $24 \mathrm{~h}$. The solution was then concentrated under reduced pressure; the yellow precipitate obtained was recrystallized from diethyl ether $\left(59 \%\right.$ yield). M.p. $106-109^{\circ} \mathrm{C}$. Found: C, 61.7; H, 5.2; N, 14.4. Calc. for $\mathrm{C}_{40} \mathrm{H}_{40} \mathrm{~N}_{8} \mathrm{O}_{5} \mathrm{Zn}, \mathrm{C}, 61.2 ; \mathrm{H}, 5.2 ; \mathrm{N}, 14.2$. IR (nujol, $\mathrm{cm}^{-1}$ ): $3362 \mathrm{br}$ $\mathrm{v}(\mathrm{H}, \mathrm{O}), 3170 \mathrm{wbr} v(\mathrm{C}-\mathrm{H}), 1560 \mathrm{sbr}, 1376 \mathrm{vs} v(\mathrm{COO}), 298 \mathrm{vs} v(\mathrm{Zn}-\mathrm{O})$. ${ }_{\mathrm{H}} \mathrm{NMR}\left(\mathrm{CDCl}_{3}\right): \delta, 1.9(\mathrm{br}, 2 \mathrm{H}, H, \mathrm{O})$, 2.01 (s, 6H, CH; COO), 6.97 (br, 4H, H-5), 7.10-7.34 (m, 12 H, $\left.\mathrm{C}_{6} H_{5}\right), 7.35$ (br, 4H, H-2), 7.50-7.70 (m, 8H, $\left.\mathrm{C}_{6} \mathrm{H}_{5}\right), 13.5(\mathrm{br}, 4 \mathrm{H}, \mathrm{N} H)$.

/(4-pheny/imidazole ${ }_{4} \mathrm{Zn}\left(\mathrm{CF}_{3} \mathrm{COO}\right)_{2} /\left(\mathrm{H}_{2} \mathrm{O}\right)(17)$. 4-phenylimidazole $(0.579 \mathrm{~g}, 4.0 \mathrm{mmol})$ was added to an ethanol solution $(30 \mathrm{ml})$ of $\mathrm{Zn}\left(\mathrm{CF}_{3} \mathrm{COO}\right)_{2}(0.291 \mathrm{~g}, 1.0 \mathrm{mmol})$. The reaction was stirred at room temperature for $24 \mathrm{~h}$. The solution was then concentrated under reduced pressure; the oily residue obtained was recrystallized from diethyl ether $/ \mathrm{CH}, \mathrm{Cl},\left(58 \%\right.$ yield). M.p. $154-157^{\circ} \mathrm{C}$. Found: $\mathrm{C}, 55.3 ; \mathrm{H}, 3.7 ; \mathrm{N}, 12.9$. Calc. for $\mathrm{C}_{40} \mathrm{H}_{34} \mathrm{~F}_{6} \mathrm{~N}_{8} \mathrm{O}_{5} \mathrm{Zn}, \mathrm{C}, 56.1 ; \mathrm{H}, 3.9 ; \mathrm{N}, 12.7$. IR (nujol, $\mathrm{cm}^{-1}$ ): $3134 \mathrm{w}, 3113 \mathrm{~m}, 3058 \mathrm{~m}$ v(C-H), $1654 \mathrm{vsbr}$, $1377 v s v(C O O), 1125 v s b r v(C-F), 266 \mathrm{~m}, 276 \mathrm{~m} v(\mathrm{Zn}-\mathrm{O})+v(\mathrm{Zn}-\mathrm{N})$. ' $\mathrm{H}$ NMR (Acetone): $\delta, 5.0(\mathrm{br}, 2 \mathrm{H}$, $\left.H_{2} \mathrm{O}\right), 8.50-8.70\left(\mathrm{~m}, 12 \mathrm{H}, \mathrm{C}_{6} H_{5}\right), 8.88(\mathrm{~s}, 4 \mathrm{H}, H-5), 9.04\left(\mathrm{~d}, 8 \mathrm{H}, \mathrm{C}_{6} H_{5}\right), 9.36(\mathrm{~s}, 4 \mathrm{H}, H-2)$. Cond. (Acetone, conc. $\left.1.02 \times 10^{-1} \mathrm{M}\right):$ ) : $\Lambda_{\mathrm{m}} 6.8 \Omega^{1} \mathrm{~cm}^{-} \mathrm{mol}^{1}$.

J(4-pheny/inidazole $)_{3} \mathrm{Zn}\left(\mathrm{CF}, \mathrm{SO}_{3}\right)_{2} /(\mathbf{1 8})$. A diethyl ether solution $(20 \mathrm{ml})$ of $\mathrm{Zn}\left(\mathrm{CF} \quad{ }_{3} \mathrm{SO}_{3}\right)_{2}(0.363 \mathrm{~g}, 1.0$ $\mathrm{mmol})$ was added to a dichloromethane solution $(30 \mathrm{ml})$ of 4 -phenylimidazole $(0.865 \mathrm{~g} .6 .0 \mathrm{mmol})$. The reaction was stirred at room temperature for $24 \mathrm{~h}$. The solution was then concentrated under reduced pressure; the colorless precipitate obtained was recrystallized from $\mathrm{CH}_{2} \mathrm{Cl}$ /petroleum ether ( $33 \%$ yield). M.p. 92-96 ${ }^{\circ} \mathrm{C}$. Found: $\mathrm{C}, 48.5$; H. 3.4; N. 11.9. Calc. for $\mathrm{C}_{38} \mathrm{H}_{3}, \mathrm{~F}_{6} \mathrm{~N}_{8} \mathrm{O}_{6} \mathrm{~S}, \mathrm{Zn} . \mathrm{C}, 49.0 ; \mathrm{H} .3 .7:$ N. 13.3 . IR (nujol, $\left.\mathrm{cm}^{-1}\right): 3144 \mathrm{mbr} v(\mathrm{C}-\mathrm{H}), 1302 \mathrm{mbr}, 635 \mathrm{sbr}, 514 \mathrm{mbr} \vee\left(\mathrm{SO}_{3}\right), 1155 \mathrm{~m}$ V(C-F). ${ }^{1} \mathrm{H}$ NMR (Acetone): $\delta, 7.30-7.50$ (m, $\left.12 \mathrm{H}, \mathrm{C}_{6} H_{5}\right), 7.76\left(\mathrm{~d}, 8 \mathrm{H}, \mathrm{C}_{6} H_{5}\right), 7.81,(\mathrm{~s}, 4 \mathrm{H}, H-5), 8.55(\mathrm{~s}, 4 \mathrm{H}, H-2)$.

/(4-phenylinidazole $)_{0} Z n\left(B F_{4}\right), /(H, O)(19)$. Compound 19 was prepared similarly to compound 17 by using $\mathrm{Zn}\left(\mathrm{BF}_{4}\right)_{2}$. Recrystallized from diethyl ether $\left(60 \%\right.$ yield). M.p. $166-170^{\circ} \mathrm{C}$. Found: C, $57.8 ; \mathrm{H}, 4.5 ; \mathrm{N}, 15.0$. Calc. for $\mathrm{C}_{54} \mathrm{H}_{50} \mathrm{~B}_{2} \mathrm{~F}_{8} \mathrm{~N}_{12}, \mathrm{OZn}, \mathrm{C}, 57.3 ; \mathrm{H}, 4.5 ; \mathrm{N}, 15.1$. IR (nujol, $\mathrm{cm}^{-1}$ ): $3608 \mathrm{mbr}, 3318 v \mathrm{vbr} v(\mathrm{H}, \mathrm{O}), 3128 \mathrm{mbr}$ $\mathrm{v}(\mathrm{C}-\mathrm{H}), 1126 \mathrm{vs}, 1028 \mathrm{vsb}, 966 \mathrm{~s} .755 \mathrm{~s}, 518 \mathrm{~s} v\left(\mathrm{BF}_{4}\right), 205 \mathrm{~s} v(\mathrm{Zn}-\mathrm{N}), 279 \mathrm{~m}, 247 \mathrm{~m}, 227 \mathrm{~m} \mathrm{v}(\mathrm{Zn}-\mathrm{F})$. H NMR (Acetone): $\delta .7 .20-$ $7.50\left(\mathrm{~m} .18 \mathrm{H}, \mathrm{C}_{6} H_{5}\right), 7.71(\mathrm{~s}, 6 \mathrm{H}, H-5), 7.77\left(\mathrm{~d}, 12 \mathrm{H}, \mathrm{C}_{6} H_{5}\right), 8.26(\mathrm{~s}, 6 \mathrm{H}, H-2) .9 .9$ (br, 6H, NH).

/(4-methylimidazole) ${ }_{2} \mathrm{ZnCl}, /\left(\mathrm{H}_{2} \mathrm{O}\right)_{0.5} ;(\mathbf{2 0})$. A diethyl ether suspension $(20 \mathrm{ml})$ of $\mathrm{ZnCl}_{2}(0.136 \mathrm{~g}, 1.0 \mathrm{mmol})$ was added to an ethanol solution $(30 \mathrm{ml})$ of 4 -methylimidazole $(0.328 \mathrm{~g}, 4.0 \mathrm{mmol})$. The reaction was stirred at room temperature for $24 \mathrm{~h}$. The solution was then concentrated under reduced pressure; the oily residue obtained was washed from diethyl ether $(50 \%$ yield). Found: C, 36.8: H. 5.6: N. 21.5. Calc. for $\mathrm{C}_{12} \mathrm{H}_{10} \mathrm{Cl}_{2} \mathrm{~N}_{6} \mathrm{O}_{0 .}: \mathrm{Zn}, \mathrm{C} .37 .1 ; \mathrm{H}, 5.8 ; \mathrm{N}, 21.5$. IR (nujol, $\left.\mathrm{cm}^{-1}\right): 3380 \mathrm{mbr} v\left(\mathrm{H}_{2} \mathrm{O}\right)$, 3133mbr v(C-H), 303s v $(\mathrm{Zn}-$ Cl), $227 \mathrm{w} v(\mathrm{Zn}-\mathrm{N}) .200 \mathrm{~s} v(\mathrm{~N}-\mathrm{N})$. HNMR(Aatax): $\delta, 2.23$ (s, $9 \mathrm{H}, \mathrm{CH}$ ). 6.90 (s. $3 \mathrm{H}, H-5) .7 .86$ (s, $3 \mathrm{H} . H-2)$.

/(4-methylimidazole $)_{+} Z n B r_{3} /\left(H_{2} O\right)_{1.5}(21)$. A diethyl ether suspension $(20 \mathrm{ml})$ of $\mathrm{ZnBr}_{2}(0.225 \mathrm{~g} .1 .0 \mathrm{mmol})$ was added to a $\mathrm{CH}_{2} \mathrm{Cl}_{2}$ solution $(30 \mathrm{ml})$ of 4 -methylimidazole $(0.328 \mathrm{~g}, 4.0 \mathrm{mmol})$. The reaction was stirred at room temperature for $24 \mathrm{~h}$. The solution was then concentrated under reduced pressure; the oily residue obtained was washed from $\mathrm{CH}_{2} \mathrm{Cl}_{2} /$ diethyl ether $(40 \%$ yield). Found: $\mathrm{C}, 33.0: \mathrm{H}, 4.7: \mathrm{N}$. 19.1. Calc. for $\mathrm{C}_{16} \mathrm{H}_{27} \mathrm{Br}_{2} \mathrm{~N}_{8} \mathrm{O}_{1,5} \mathrm{Zn}$. C. 33.3: H, 4.7: N. 19.4. IR (nujol. $\mathrm{cm}^{-1}$ ): $3129 \mathrm{mbr} v(\mathrm{C}-\mathrm{H}) .226 \mathrm{~m} v(\mathrm{Zn}-\mathrm{Br}) .201 \mathrm{w} v(\mathrm{~N}-$ N). ${ }^{1} \mathrm{H}$ NMR (Acetone): $\delta, 2.24\left(\mathrm{~s}, \mathrm{I} 2 \mathrm{H}, \mathrm{CH}_{3}\right), 5.0$ (br, $\left.3 \mathrm{H}, \mathrm{H}_{2} \mathrm{O}\right), 6.89$ (s. $4 \mathrm{H}, \mathrm{H}-5$ ), 7.84 (s. $4 \mathrm{H} . \mathrm{H}-2$ ). 
/(4-nethylimidazole $)_{3} \mathrm{Zn}\left(\mathrm{NO}_{3}\right)_{2} /\left(\mathrm{H}_{2} \mathrm{O}\right)_{1.5^{\prime}}$ (22). Compound 22 was prepared similarly to compound 20, by using $\mathrm{Zn}\left(\mathrm{NO}_{3}\right)$ ). The oil obtained was washed from $n$-pentane $(40 \%$ yield $)$. Found: $\mathrm{C}, 31.1 ; \mathrm{H}, 4.6 ; \mathrm{N}, 24.2$. Calc. for $\mathrm{C}_{12} \mathrm{H}_{21} \mathrm{~N}_{8} \mathrm{O}_{7.5} \mathrm{Zn}, \mathrm{C}, 31.0 ; \mathrm{H}, 4.6 ; \mathrm{N}, 24.1$. IR (nujol, $\mathrm{cm}^{-1}$ ): $332 \mathrm{lwbr} v(\mathrm{H}, \mathrm{O}), 3144 \mathrm{wbr} v(\mathrm{C}-\mathrm{H})$, $1750 \mathrm{vw}, 1734 \mathrm{vw} \mathrm{v}_{1}+\mathrm{v}_{4}\left(\mathrm{NO}_{3}\right), 1376 \mathrm{vs} v\left(\mathrm{NO}_{3}\right), 286 \mathrm{w}, 274 \mathrm{w} v(\mathrm{Zn}-\mathrm{O}), 236 \mathrm{~m}, 206 \mathrm{~s} v(\mathrm{Zn}-\mathrm{N}), 200 \mathrm{w} v(\mathrm{~N}-\mathrm{N})$. ${ }^{1} \mathrm{H}$ NMR (Acetone): $\delta, 2.27\left(\mathrm{~s}, 9 \mathrm{H}, \mathrm{CH}_{3}\right), 3.20(\mathrm{br}, 3 \mathrm{H}, H, \mathrm{O}), 7.01(\mathrm{~s}, 3 \mathrm{H}, H-5), 8.10$ (s, 3H, $\left.H-2\right)$.

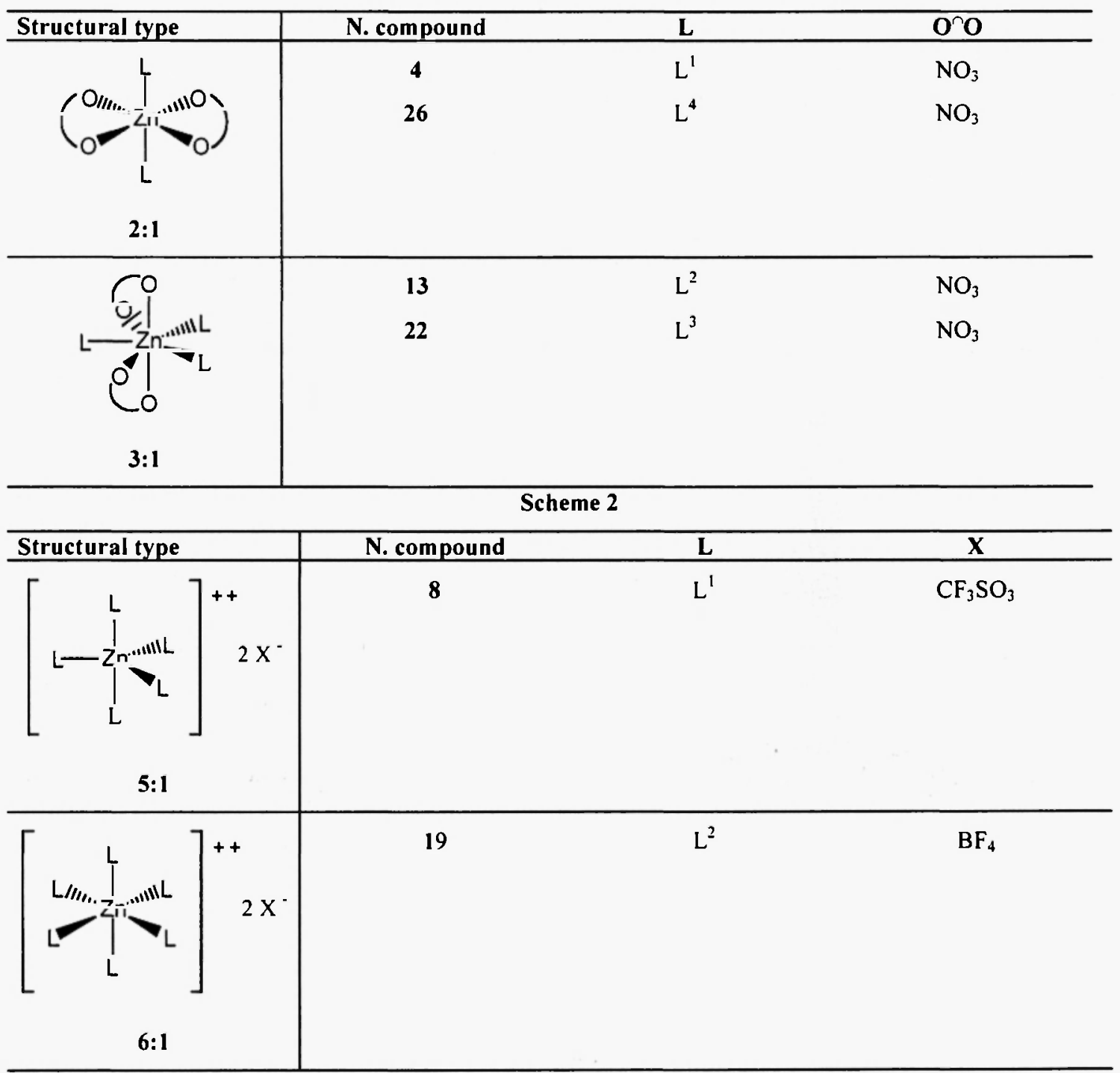

\section{Scheme 3}

/(2-phenylimidazole $)_{2} \mathrm{ZnCl} /\left(\mathrm{H}_{2} \mathrm{O}\right)_{0.5}(23) . \mathrm{ZnCl}_{2}(0.136 \mathrm{~g}, 1.0 \mathrm{mmol})$ was added to a diethyl ether solution $(30 \mathrm{ml})$ of 2-phenylimidazole $(0.579 \mathrm{~g}, 4.0 \mathrm{mmol})$. The reaction was stirred at room temperature for $24 \mathrm{~h}$. A colorless precipitate was formed, which was filtered off and washed with diethyl ether $(2 \times 5 \mathrm{ml})(40 \%$ yield). M.p. $229-232^{\circ} \mathrm{C}$. Found: $\mathrm{C}, 49.9 ; \mathrm{H}, 3.9 ; \mathrm{N}, 12.9$. Calc. for $\mathrm{C}_{18} \mathrm{H}_{17} \mathrm{Cl}_{2} \mathrm{~N}_{4} \mathrm{O}_{05} \mathrm{Zn}, \mathrm{C}, 49.6 ; \mathrm{H}, 3.9 ; \mathrm{N}$, 12.9. IR (nujol, $\mathrm{cm}^{-1}$ ): $3243 \mathrm{mbr} v\left(\mathrm{H}_{2} \mathrm{O}\right), 3127 \mathrm{w}, 3051 \mathrm{vw} v(\mathrm{C}-\mathrm{H}), 321 \mathrm{~m}, 289 \mathrm{vs} \mathrm{v}(\mathrm{Zn}-\mathrm{Cl}), 235 \mathrm{~m} v(\mathrm{Zn}-\mathrm{N})$. ' $\mathrm{H}$ NMR (Acetone): $\delta, 2.97\left(\mathrm{br}, 1 \mathrm{H}, H_{2} \mathrm{O}\right), 7.15-7.50\left(\mathrm{~m}, 10 \mathrm{H}, \mathrm{C}_{6} H_{5}, H-4\right.$ and $\left.H-5\right), 7.63\left(\mathrm{~d}, 4 \mathrm{H}, \mathrm{C}_{6} H_{5}\right), 12.0(\mathrm{br}$, $2 \mathrm{H}, \mathrm{N} H)$.

/(2-phenylimidazole) $\mathrm{ZnBr}_{2} /(24)$. A diethyl ether suspension $(20 \mathrm{ml})$ of $\mathrm{ZnBr} \quad{ }_{2}(0.225 \mathrm{~g}, 1.0 \mathrm{mmol})$ was added to a $\mathrm{CH}_{2} \mathrm{Cl}_{2}$ solution $(30 \mathrm{ml})$ of 2 -phenylimidazole $(0.865 \mathrm{~g}, 6.0 \mathrm{mmol})$. The reaction was stirred at room temperature for $24 \mathrm{~h}$. The solution was then concentrated under reduced pressure; the colorless precipitate obtained was recrystallized from $\mathrm{CH}_{2} \mathrm{Cl}$, $\left(61 \%\right.$ yield). M.p. $95-98^{\circ} \mathrm{C}$. Found: $\mathrm{C}, 44.1 ; \mathrm{H}, 3.1 ; \mathrm{N}$, 11.2. Calc. for $\mathrm{C}_{18} \mathrm{H}_{16} \mathrm{Br}, \mathrm{N}_{4} \mathrm{Zn}, \mathrm{C}, 44.6 ; \mathrm{H}, 3.4 ; \mathrm{N}, 11.6$. IR (nujol, $\mathrm{cm}^{-1}$ ): $3127 \mathrm{~m} \mathrm{v}(\mathrm{C}-\mathrm{H}), 215 v s \mathrm{~V}(\mathrm{Zn}-\mathrm{Br}$ ), $244 \mathrm{~s}, 229 \mathrm{w} v(\mathrm{Zn}-\mathrm{N})$. 'H NMR (Acetone): $\delta, 7.10-7.60\left(\mathrm{~m}, 10 \mathrm{H}, \mathrm{C}_{6} H_{5}, H-4\right.$ and $\left.H-5\right), 7.65\left(\mathrm{~d}, 4 \mathrm{H}, \mathrm{C}_{6} H_{5}\right)$, $12.0(\mathrm{br}, 2 \mathrm{H}, \mathrm{N} H)$. 
I(2-phenylımidazole) $\mathrm{ZnI}_{2} \mathrm{I}\left(\mathrm{H}_{2} \mathrm{O}\right)_{1.5}$ (25). A diethyl ether suspension $(20 \mathrm{ml})$ of $\mathrm{ZnI}_{2}(0.319 \mathrm{~g}, 1.0 \mathrm{mmol})$ was added to a $\mathrm{CH}_{2} \mathrm{Cl}_{2}$ solution $(30 \mathrm{ml})$ of 2 -phenylimidazole $(0.579 \mathrm{~g}, 4.0 \mathrm{mmol})$. The reaction was stirred at room temperature for $24 \mathrm{~h}$. The solution was then concentrated under reduced pressure; the yellow precipitate obtained was recrystallized from $\mathrm{CH}_{2} \mathrm{Cl}_{2} /$ diethyl ether $\left(61 \%\right.$ yield). M.p. $112-115^{\circ} \mathrm{C}$. Found: $\mathrm{C}$, 46.9; $\mathrm{H}, 3.8 ; \mathrm{N}, 12.1$. Calc. for $\mathrm{C}_{36} \mathrm{H}_{35} \mathrm{I}_{2} \mathrm{~N}_{8} \mathrm{O}_{1.5} \mathrm{Zn}, \mathrm{C}, 46.8 ; \mathrm{H}, 4.0 ; \mathrm{N}, 11.8$. IR (nujol, $\mathrm{cm}^{-1}$ ): $3159 \mathrm{wbr} \mathrm{v}(\mathrm{C}-\mathrm{H})$, $233 \mathrm{~m} v(\mathrm{Zn}-\mathrm{N})$. ' $\mathrm{H}$ NMR (Acetone): $\delta, 5.6(\mathrm{br}, 3 \mathrm{H}, H, \mathrm{O}), 7.0-7.6\left(\mathrm{~m}, 20 \mathrm{H}, \mathrm{C}_{6} H_{5}, H-4\right.$ and $\left.H-5\right), 7.76(\mathrm{br}, 8 \mathrm{H}$, $\left.\mathrm{C}_{6} H_{5}\right), 12.0(\mathrm{br}, 4 \mathrm{H}, \mathrm{N} H)$.

I(2-phenylimidazole) ${ }_{2} \mathrm{Zn}\left(\mathrm{NO}_{3}\right)_{2} /\left(\mathrm{H}_{2} \mathrm{O}\right)_{1} \dot{5}$ (26). Compound 26 was prepared similarly to compound 23, by using $\mathrm{Zn}\left(\mathrm{NO}_{3}\right)$, (36\% yield). M.p. $178-181^{\circ} \mathrm{C}$. Found: $\mathrm{C}, 42.5 ; \mathrm{H}, 3.8 ; \mathrm{N}, 16.6$. Calc. for $\mathrm{C}_{18} \mathrm{H}_{19} \mathrm{~N}_{6} \mathrm{O}_{7.5} \mathrm{Zn}, \mathrm{C}$, $42.1 ; \mathrm{H}, 3.8 ; \mathrm{N}, 16.5$. IR (nujol, $\left.\mathrm{cm}^{-1}\right)$ : 3539w, 3485w, 3369wbr v(H,O) $+\mathrm{v}(\mathrm{NH}), 3156 \mathrm{wbr} v(\mathrm{C}-\mathrm{H})$, $1751 \mathrm{l} w$ $\mathrm{v}_{1}+\mathrm{v}_{4}\left(\mathrm{NO}_{3}\right), 1377 \mathrm{vs} v\left(\mathrm{NO}_{3}\right), 274 \mathrm{mbr} v(\mathrm{Zn}-\mathrm{O})$. ' $\mathrm{H}$ NMR (Acetone): $\left.\delta, 3.4 \mathrm{br}, 3 \mathrm{H}, H, \mathrm{O}\right), 7.20-7.50$ ( $\mathrm{m}, 14 \mathrm{H}$, $\mathrm{C}_{6} H_{5}, H-4$ and $\left.H-5\right), 12.1(\mathrm{br}, 2 \mathrm{H}, \mathrm{N} H)$. Cond. (Acetone, conc. $\left.1.10 \times 10^{-3} \mathrm{M}\right):$ ): $\Lambda_{\mathrm{m}} 13.7 \Omega^{-1} \mathrm{~cm}^{-2} \mathrm{~mol}^{-1}$.

/(2-phenylimidazole $)_{3} \mathrm{Zn}\left(\mathrm{ClO}_{4}\right)_{2} / \mathrm{H}_{2} \mathrm{O}$ ( 27). A diethyl ether suspension $(20 \mathrm{ml})$ of $\mathrm{Zn}\left(\mathrm{ClO}{ }_{4}\right)_{2}(0.264 \mathrm{~g}, 1.0$ mmol) was added to a $\mathrm{CH}_{2} \mathrm{Cl}_{2}$ solution $(30 \mathrm{ml})$ of 2-phenylimidazole $(0.579 \mathrm{~g}, 4.0 \mathrm{mmol})$. The reaction was stirred at room temperature for $24 \mathrm{~h}$. The colorless precipitate obtained was recrystallized from $\mathrm{CH}, \mathrm{Cl} /$ diethyl ether $\left(76 \%\right.$ yield). M.p. $204-208^{\circ} \mathrm{C}$. Found: $\mathrm{C}, 45.4 ; \mathrm{H}, 3.7 ; \mathrm{N}, 11.9$. Calc. for $\mathrm{C}_{77} \mathrm{H}_{36} \mathrm{Cl}_{2} \mathrm{~N}_{6} \mathrm{O}_{9} \mathrm{Zn}, \mathrm{C}, 45.2 ; \mathrm{H}, 3.8 ; \mathrm{N}, 12.3$. IR (nujol, $\mathrm{cm}^{-1}$ ): $3252 \mathrm{sbr} v(\mathrm{H}, \mathrm{O}), 3130 \mathrm{~m}, 3060 \mathrm{w} v(\mathrm{C}-\mathrm{H})$, $1120 \mathrm{vsbr}, 1064 \mathrm{vsbr}, 1000 \mathrm{w}, 621 \mathrm{~s}, 617 \mathrm{vs} \mathrm{v}\left(\mathrm{ClO}_{4}\right), 278 \mathrm{vw}, 237 \mathrm{~m} v(\mathrm{Zn}-\mathrm{O}), 208 \mathrm{~m} v(\mathrm{~N}-\mathrm{N}),{ }^{1} \mathrm{H}$ NMR (Acetone): $\delta, 7.2-8.0$ (br, $15 \mathrm{H}, \mathrm{C}_{6} H_{5}, H-4$ and $\left.H-5\right), 9.0\left(\mathrm{br}, 6 \mathrm{H}, \mathrm{C}_{6} H_{5}\right), 12.0(\mathrm{br}, 3 \mathrm{H}, \mathrm{N} H$ ).

/(2-phenylimidazole) ${ }_{2} \mathrm{Zn}\left(\mathrm{CH}_{3} \mathrm{COO}\right)_{2} / \mathrm{H}_{2} \mathrm{O}(28)$. Compound 28 was prepared similarly to compound 27 , by using $\mathrm{Zn}\left(\mathrm{CH}_{3} \mathrm{COO}\right),\left(55 \%\right.$ yield). M.p. $192-195^{\circ} \mathrm{C}$. Found: $\mathrm{C}, 54.0 ; \mathrm{H}, 4.9 ; \mathrm{N}, 11.4$. Calc. for $\mathrm{C}_{22} \mathrm{H}_{4} \mathrm{~N}_{4} \mathrm{O}_{5} \mathrm{Zn}$, C, 54.0; H, 4.9; N, 11.4. IR (nujol, $\mathrm{cm}^{-1}$ ): 3370vwbr v(H,O), 3134vwbr, 3062vwbr v(C-H), 1560vsbr, 1377m $\mathrm{v}(\mathrm{COO}), 292 \mathrm{vsbr} v(\mathrm{Zn}-\mathrm{O})$. ' $\mathrm{H}$ NMR (Acetone): $\delta, 1.95\left(\mathrm{~s}, 6 \mathrm{H}, \mathrm{CH}_{3} \mathrm{COO}\right), 2.84\left(\mathrm{br}, 2 \mathrm{H}, \mathrm{H}_{2} \mathrm{O}\right), 7.15(\mathrm{~s}, 4 \mathrm{H}$, $H-4$ and $H-5), 7.30-7.50,\left(\mathrm{~m}, 6 \mathrm{H}, \mathrm{C}_{6} H_{5}\right), 7.99\left(\mathrm{~d}, 4 \mathrm{H}, \mathrm{C}_{6} H_{5}\right), 11.6(\mathrm{br}, 2 \mathrm{H}, \mathrm{N} H)$.

/(2-phenylimidazole $)_{4} Z n\left(C F F_{3} C O O\right)_{2} /(29)$. A diethyl ether suspension $(20 \mathrm{ml})$ of $\mathrm{Zn}\left(\mathrm{CF} \quad{ }_{3} \mathrm{COO}\right)_{2}(0.291 \mathrm{~g}$, $1.0 \mathrm{mmol})$ was added to a $\mathrm{CH}_{2} \mathrm{Cl}_{2}$ solution $(30 \mathrm{ml})$ of 2-phenylimidazole $(0.579 \mathrm{~g}, 4.0 \mathrm{mmol})$. The reaction was stirred at room temperature for $24 \mathrm{~h}$. The solution was then concentrated under reduced pressure; the colorless precipitate obtained was recrystallized from $\mathrm{CH}, \mathrm{Cl}_{2} /$ diethyl ether $(81 \%$ yield $)$. M.p. $129-132^{\circ} \mathrm{C}$. Found: $\mathrm{C}, 55.3 ; \mathrm{H}, 3.7 ; \mathrm{N}, 12.9$. Calc. for $\mathrm{C}_{40} \mathrm{H}_{32} \mathrm{~F}_{6} \mathrm{~N}_{8} \mathrm{O}_{4} \mathrm{Zn}, \mathrm{C}, 55.4 ; \mathrm{H}, 3.8 ; \mathrm{N}, 13.2$. IR (nujol, $\mathrm{cm}^{-1}$ ): $3159 \mathrm{mbr} v(\mathrm{C}-\mathrm{H}), 1652 \mathrm{mbr}, 1374 \mathrm{vs} v(\mathrm{COO}), 1154 \mathrm{vs} v(\mathrm{C}-\mathrm{F}), 272 \mathrm{~s} v(\mathrm{Zn}-\mathrm{O}), 216 \mathrm{w} v(\mathrm{Zn}-\mathrm{N})$. 'H NMR (Acetone): $\delta, 7.20-7.40\left(\mathrm{~m}, 20 \mathrm{H}, \mathrm{C}_{6} H_{5}, H-4\right.$ and $\left.H-5\right), 7.70\left(\mathrm{br}, 8 \mathrm{H}, \mathrm{C}_{6} H_{5}\right)$.

/(2-phenylimidazole) ${ }_{4} \mathrm{Zn}\left(\mathrm{CF}_{3} \mathrm{SO}_{3}\right)_{2} /(\mathbf{3 0})$. Compound 30 was prepared similarly to compound 29 , by using $\mathrm{Zn}\left(\mathrm{CF}_{3} \mathrm{SO}_{3}\right)$ ). Recrystallized from diethyl ether $\left(50 \%\right.$ yield). M.p. $128-131^{\circ} \mathrm{C}$. Found: $\mathrm{C}, 48.8 ; \mathrm{H}, 3.4 ; \mathrm{N}$, 11.9. Calc. for $\mathrm{C}_{38} \mathrm{H}_{3}, \mathrm{~F}_{6} \mathrm{~N}_{8} \mathrm{O}_{6} \mathrm{~S} 2 \mathrm{Zn}, \mathrm{C}, 49.2 ; \mathrm{H}, 3.6 ; \mathrm{N}, 11.7$. IR (nujol, $\mathrm{cm}^{-1}$ ): 3147wbr, 3059vw v(C-H),

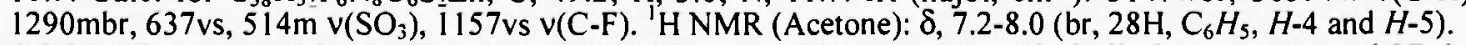

2.2.31. /(2-phenylimidazole). $Z n\left(B F_{4}\right)_{2} /(31)$. Compound 31 was prepared similarly to compound 27 , by using $\mathrm{Zn}\left(\mathrm{BF}_{4}\right),\left(27 \%\right.$ yield). M.p. $269-272^{\circ} \mathrm{C}$. Found: $\mathrm{C}, 48.5 ; \mathrm{H}, 3.6 ; \mathrm{N}, 12.9$. Calc. for $\mathrm{C}_{7} \mathrm{H}_{24} \mathrm{~B}_{2} \mathrm{~F}_{8} \mathrm{~N}_{6} \mathrm{Zn}, \mathrm{C}$, $48.9 ; \mathrm{H}, 3.6 ; \mathrm{N}$, 13.3. IR (nujol, $\mathrm{cm}^{-1}$ ): $3299 \mathrm{vsbr}, 3138 \mathrm{mbr}, 3062 \mathrm{w} \mathrm{v}(\mathrm{C}-\mathrm{H}), 1121 \mathrm{vs}, 1052 \mathrm{vsbr}, 1014 \mathrm{~s}, 778 \mathrm{vs}$, $522 \mathrm{~m} v\left(\mathrm{BF}_{4}\right), 202 \mathrm{~s} v(\mathrm{Zn}-\mathrm{N}), 280 \mathrm{w}, 250 \mathrm{~m}, 222 \mathrm{w} v(\mathrm{Zn}-\mathrm{F})$. ' $\mathrm{H}$ NMR (Acetone): $\delta, 7.0-7.8\left(\mathrm{~m} \mathrm{br}, 21 \mathrm{H}, \mathrm{C}_{6} H_{5}\right.$, $H-4$ and $H-5), 12.1(\mathrm{br}, 3 \mathrm{H}, \mathrm{N} H)$. Cond. (Acetone, conc. $\left.\left.1.02 \times 10^{-3} \mathrm{M}\right):\right): \Lambda_{\mathrm{m}} 176.7 \Omega^{-1} \mathrm{~cm}^{2} \mathrm{~mol}^{-1}$.

\section{Results and Discussion}

\section{Synthesis of the $\mathrm{Zn}(\mathrm{ll})$ complexes}

Benzimidazole $\left(\mathrm{L}^{1}\right)$, 4-phenylmidazole $\left(\mathrm{L}^{2}\right)$, 4-methylimidazole $\left(\mathrm{L}^{3}\right)$ or 2-phenylimidazole $\left(\mathrm{L}^{4}\right)$ react in ethanol, diethyl ether or ethanol/diethyl ether solution/suspension with $\mathrm{ZnX}$, acceptors $(\mathrm{X}=\mathrm{Cl}, \mathrm{Br}, \mathrm{l}$, $\mathrm{NO}_{3}, \mathrm{ClO}_{4}, \mathrm{CH}_{3} \mathrm{COO}, \mathrm{CF}_{3} \mathrm{COO}, \mathrm{CF}_{3} \mathrm{SO}_{3}$ or $\mathrm{BF}_{4}$ ) affording derivatives 1-31 (schemes 1, 2 and 3):

$$
\begin{array}{r}
n \mathrm{~L}+m \mathrm{ZnX}{ }_{2}\left(\mathrm{H}_{2} \mathrm{O}\right)_{\mathrm{x}} \longrightarrow(\mathrm{L})_{n}\left(\mathrm{ZnX}_{2}\right)_{m}\left(\mathrm{H}_{2} \mathrm{O}\right)_{\mathbf{x}} \\
\quad(n=2,3,4,5 \text { or } 6 ; m=1 ; x=0.5,1,1.5 \text { or } 2)
\end{array}
$$

Some of these zinc(II) complexes retain or absorb solvent molecules. Derivatives of 4 methylimidazole (20-22) are amorphous below $30^{\circ} \mathrm{C}$ and oils up to $30^{\circ} \mathrm{C}$, whereas all the other compounds are air stable microcrystalline solids with sharp melting points (Experimental Section). They are generally soluble in DMSO, enough soluble in acetone and acetonitrile, poor soluble in chlorinated solvents such as chloroform and dichloromethane, insoluble in diethyl ether, alcohols and water. The solubility is function of the counterion (the dihalide and tetrafluoroborato derivatives are less soluble of the nitrato, perchlorato, and acetato complexes) and primarily of the ligand (the presence of a phenyl group generally increases the solubility of the complexes.

The derivative $\left[\left(\mathrm{L}^{\prime}\right)_{4} \mathrm{Zn}\left(\mathrm{CF}_{3} \mathrm{SO}_{3}\right)_{2}\right]$ (7) was obtained from the reaction of benzimidazole ( $\left.\mathrm{L}^{\prime}\right)$ with $\mathrm{Zn}\left(\mathrm{CF}_{3} \mathrm{SO}_{3}\right)_{2}$, in diethyl ether, using a $4 / 1$ ligand/metal ratio, whereas the derivative $\left[\left(\mathrm{L}^{\prime}\right)_{5} \mathrm{Zn}\left(\mathrm{CF}_{3} \mathrm{SO}_{3}\right)_{2}\right](8)$ was obtained from the reaction of benzimidazole with $\mathrm{Zn}\left(\mathrm{CF}_{3} \mathrm{SO}_{3}\right)_{2}$, in ethanol, using a $6 / 1$ ligand/metal ratio; analogously, the derivative $\left[\left(\mathrm{L}^{2}\right)_{3} \mathrm{Zn}\left(\mathrm{CH}_{3} \mathrm{COO}\right)_{2}\right](15)$ was obtained from the reaction of 4 phenylimidazole $\left(\mathrm{L}^{2}\right)$ with $\mathrm{Zn}\left(\mathrm{CH}_{3} \mathrm{COO}\right)_{2}$, in dichloromethane/diethyl ether, whereas the derivative 
$\left[\left(\mathrm{L}^{2}\right)_{4} \mathrm{Zn}\left(\mathrm{CH}_{3} \mathrm{COO}\right)_{2}\right]$ (16). was obtained from the reaction of 4-phenylimidazole with $\mathrm{Zn}\left(\mathrm{CH}_{3} \mathrm{COO}\right)_{2}$ in ethanol/diethyl ether, using the same ligand/metal ratio.

IR data

Selected IR and far-IR data of derivatives 1-31 are reported in the Experimental Section. The spectra are recorded in Nujol mull.

In the region $3200-3000 \mathrm{~cm}^{-1}$ we have found the $v(\mathrm{C}-\mathrm{H})$ of azoles always shifted to higher frequencies (at least $50 \mathrm{~cm}^{-1}$ ) with respect to the $\mathrm{v}(\mathrm{C}-\mathrm{H})$ of aromatic rings and generally shifted of $5-10 \mathrm{~cm}^{-1}$ with respect to the corresponding vibrations in the free ligands. In the region $1650-1500 \mathrm{~cm}^{-1}$ the $v\left(C^{-1} N\right)$ and $\mathrm{v}\left(\mathrm{C}^{-}-\mathrm{C}\right)$ absorptions, while the bending vibrations typical of the ligands and the Whiffen absorptions $\mathrm{s}^{30,-1}$ are observed in the region $600-500 \mathrm{~cm}^{-1}$; no changes have been observed with respect to the same absorptions in the free ligands.

The di-halo-metal adducts 1-3, 10-12, 20, 21 and 23-25 show two $\mathrm{M}-\mathrm{X}$ stretching vibrations as expected for a pseudo-tetrahedral $\left(\mathrm{C}_{2 v}\right.$ symmetry) structure in the case of four-coordinate $1,2,23$ and 24 or for a cis- $\mathrm{X}_{2}$ arrangement in the case of five- or six-coordinate 3, 10-12, 20, 21 and 25 . In our case, the assignments of the $\mathrm{M}$-halogen symmetric and asymmetric stretching vibrations are red-shifted on going from $\mathrm{M}-\mathrm{Cl}\left(310-280 \mathrm{~cm}^{-1}\right)$ to $\mathrm{M}-\mathrm{Br}\left(250-225 \mathrm{~cm}^{-1}\right)$ and to $\mathrm{M}-\mathrm{I}\left(230-185 \mathrm{~cm}^{-1}\right)$. $^{32}$ The medium absorptions in the range $250-200 \mathrm{~cm}^{-1}$ can be assigned to $v(\mathrm{M}-\mathrm{N})$ stretching modes: ${ }^{32}$ in the di-chloro- and di-iodo-zinc derivatives the $v(M-N)$ modes can be assigned without much ambiguity, but for the di-bromo-zinc derivatives they lie close to the $\mathrm{v}(\mathrm{Zn}-\mathrm{Br})$ region and coupling must be appreciable.

The IR spectra of zinc-nitrate derivatives $(4,13,22$ and 26) are in accordance with the stoichiometries and the structures proposed. The derivatives containing $3 \mathrm{~N}$-ligands and 2 nitrato groups (13 and 22) should have a seven-coordinate $\mathrm{N}_{3} \mathrm{ZnO}_{4}$ core, reached through the chelation of both the nitrato groups. ${ }^{4-36}$ Derivatives 4 and 26 , with a ligand-metal ratio $2: 1$, possess an octahedral geometry in the solid state with both the counterions bidentate; the IR data (the $v_{1}-v_{4}$ stretching modes, the combination band $v_{1}+$ $v_{4}$ ) are in accordance with this evidence. ${ }^{35.36}$ In no case the spectra are in accordance with a ionic formulation of the nitrato group. ${ }^{.6}$

The perchlorato derivatives $(5,14$ and 27$)$ exhibit two different trends: $\ln 14$ and 27 the ligands $\mathrm{L}^{2}$ and $\mathrm{L}^{4}$ are not able to completely substitute the perchlorato ion in the coordination sphere of the metal; on the other hand the derivative of $\mathrm{L}^{\prime}(5)$ is ionic in the solid state $\left(\mathrm{N}_{4} \mathrm{Zn}^{+2}\right)$ and shows a broad absorption at 1094 $\mathrm{cm}^{-1}$ and a sharp and strong band at $622 \mathrm{~cm}^{-1}$, both typical of a XY $\mathrm{XY}_{4}$ ionic group. ${ }^{29}$

On the basis of IR data we can hypothesize a six-coordinate geometry for derivative 9 in the solid state, with both $\mathrm{BF}_{4}{ }^{-}$groups unidentate: in fact $v_{3}$ mode splits into two broad bands $\left(1170\right.$ and $\left.1154 \mathrm{~cm}^{-1}\right)$. We observed also two bands at 768 and $722 \mathrm{~cm}^{-1}$ due to $v_{1}$ and $v_{4}$ respectively. ${ }^{37 .}{ }^{38}$ Moreover, the absorptions at $245-280 \mathrm{~cm}^{-1}$ could be tentatively assigned to $v(\mathrm{Zn}-\mathrm{F})$ modes. In the spectrum of 31 we observe a strong broad absorption in the region $980-1150 \mathrm{~cm}^{-1}$, several broad bands in the range $680-780 \mathrm{~cm}^{-1}$ and absorptions at $250,280 \mathrm{~cm}^{-1}$ that could be tentatively assigned to $\mathrm{v}(\mathrm{Zn}-\mathrm{F})$ modes, in accordance with a penta-coordinate geometry in the solid state, with both $\mathrm{BF}_{4}{ }_{4}$ groups unidentate. However in acetone the derivative $\mathbf{3 1}$ shows a conductance value typical of $1: 1$ ionic species (see Experimental Section), suggesting that in solution this compound dissociates only one of the two $\mathrm{BF}_{4}{ }_{4}$ groups. On the other hand 19 is ionic in the solid state with the $\mathrm{BF}_{4}{ }_{4}$ groups likely linked through hydrogen bond to the $\mathrm{H}_{2} \mathrm{O}$ molecule.

In the case of carboxylate derivatives it is generally accepted ${ }^{39}$ that it is possible to distinguish between ionic, unidentate, chelating bidentate or bridging bidentate groups on the basis of $\Delta$ value [where $\Delta=$ $\left.v_{a}(\mathrm{COO})-v_{s}(\mathrm{COO})\right]$, the following trend being found:

$$
\Delta_{\text {unidentate }}>\Delta_{\text {lonic }}>\Delta_{\text {bndging bidentale }}>\Delta_{\text {chelating bidentare }}
$$

where $\Delta_{\text {ionic }}$ is $c a$. $160-170 \mathrm{~cm}^{-1}$ for acetates and $c a .220-230 \mathrm{~cm}^{-1}$ for trifluoroacetates ${ }^{37}$. In the derivatives 6 , 15,16 and 28 we predict a unidentate coordinating behaviour of $\mathrm{CH}_{3} \mathrm{COO}^{-}, \Delta$ being in the range $180-235 \mathrm{~cm}^{-}$ . In the spectra of tri-fluoroacetates 17 and 29 the $\Delta$ values are 277 and $278 \mathrm{~cm}^{-1}$ respectively, in accordance with a unidentate formulation of the $\mathrm{CF}_{3} \mathrm{COO}^{-}$groups. In all carboxylato derivatives we have also found a new band in the range $266-298 \mathrm{~cm}^{-1}$ likely due to $v(\mathrm{M}-\mathrm{O})$.

The $\mathrm{CF}_{3} \mathrm{SO}_{3}$ group in derivatives $7,8,18$ and 30 shows a spectroscopic behaviour typical of a ionic outer-sphere group. The vibrations of the $\mathrm{SO}_{3}$ and $\mathrm{CF}_{3}$ groups are similar to those observed in the spectra of analogous derivatives of bis(pyrazolyl)alkanes. ${ }^{40}$

${ }^{\prime} \mathrm{H}$ and ${ }^{\prime 3} \mathrm{C} N M R$ data

The proton NMR spectra of derivatives 1-31 have been recorded in deuterated chloroform or acetone (see Experimental Section). The choice of the solvent was dictated by the solubility of the compounds.

The signals of the azole ring protons of the ligands have always been found shifted to lower field upon coordination. The same trend has been observed for the proton signals of the R groups bonded in the 2- 
and 4-positions of the imidazole ring $(\mathrm{R}=\mathrm{Me}$ or $\mathrm{Ph})$ and for the condensed ring in $\mathrm{L}^{\prime}$ derivatives. However, the deshielding observed is attenuated at positions remote from the metal. The $\Delta$ observed is likely due to a $\sigma$-charge donation from the $\mathrm{N}$-donor to zinc(II) acceptor and is evidence of the existence of the complexes in solution. The $\Delta$ value is also function of the counterion: for example the greatest $\Delta$ values are observed in the zinc(II)derivatives containing good leaving groups $\left(\mathrm{ClO}_{4}, \mathrm{BF}_{4}\right.$ and $\left.\mathrm{CF}_{3} \mathrm{SO}_{3}\right)$.

Also if one would expect in many of these compounds the existence in solution of more than one isomer, or the presence of not equivalent ligands, in all the ' $H$ NMR spectra of 1-31 we found only one set of signals (in some cases the risonances are large, $c a .1 \mathrm{ppm}$ ) for each equivalent proton group. This is likely due to a rapid exchange between free and bound ligand or between different metal coordination sites. This rapid exchange is operative also at low temperature.

The spectra of derivatives containing imidazoles with aryl substituents often present signals shifted to higher field with respect to the analogous ligands with alkyl substituents. This is probably due to the nearness of these protons to the deshielding cone of the aromatics rings. ${ }^{26.27}$

The ${ }^{13} \mathrm{C}$ NMR spectra have been recorded in deuterated acetone only for selected compounds $(\mathbf{1}, \mathbf{5}$, 10 and 11) due to the poor solubility of most of adducts obtained (see Experimental Section).

In the benzimidazole complexes 1 and $\mathbf{5}$ the signals of the azole ring carbons have always been found shifted to lower field upon coordination and the same trend has been observed for the carbon signals of the condensed ring. It is very interesting to note that some carbon signals of the condensed ring, broad in the spectrum of the ligand, are sharp in the corresponding zinc derivatives. This is likely due to the fact that the concomitant prototropy and metallotropy, generally observed in azole complexes, ${ }^{20,41-43}$ is slow with respect to the prototropy operating in the ligand.

Finally in the 4-phenylimidazole complexes 10 and 11 the signals of the azole ring carbons have also been found shifted to lower field upon coordination, while no change has been found for the carbon signals of the aromatic ring. In all complexes the carbon signals generally are broad likely due to a fluxional behaviour operating in solution, which makes equivalent all the ligands coordinated.

\section{Conductivity data}

Conductivity measurements have been performed in dichloromethane or acetone solution for several derivatives (see Experimental Section).

Derivatives 10 and 11 are non electrolytes. Derivatives 4, 12,13, 17 and 26 for which a non-ionic structure has been observed in the solid state (see the IR data Section) show conductance values in acetone, in accordance with only partial ionic dissociation in this solvent. The dissociation seems to be dependent upon concentration of the solutions.

Finally, in the case of 31 ionic species can be postulated in solution, with the counterions $\mathrm{BF}_{4}{ }_{4}$ out of the inner coordination sphere of the metal.

\section{Conclusions}

The combined evidence from measurements carried out in-solid state and in solution (medium and far IR, conductivity, NMR spectra) gives here a complete picture about the interaction of imidazole-type ligands and $\mathrm{Zn}(\mathrm{II})$ acceptors.

The ligand/metal ratio $m: n$ has been found to vary in a wide range, so that different compounds can be obtained depending on:

I. nature of the counter-ion: in some cases it is possible to obtain, with the same ligand and in the same reaction conditions, derivatives characterized by different stoichiometries, geometries and coordination numbers.

2. steric hindrance and basicity of the ligand: for example from the interaction of the sterically hindered benzimidazole and 2-phenylimidazole with $\mathrm{ZnCl}$, we generally obtained derivatives with a 2:1 ligand/metal ratio; instead the ligands 4-rnethylimidazole and 4-phenylimidazole, without direct steric demand on the metal, gives also 3:1 adducts.

3. reaction conditions (solvent, temperature, ligand to metal ratio employed) which can help to modify structures and properties of complexes

Several interesting trends emerge: in our conditions the dichloride and dibromide complexes give generally the adducts with lower ligand/metal ratio (2:1 and $3: 1)$; only from the reaction of diiodozinc acceptors with $L^{1}, L^{2}$ and $L^{4}$ we have obtained compounds with $4: 1$ ligand/metal ratio. A pseudooctahedral 6:1 adduct $\left(\mathrm{MN}_{6}\right)$ is found only with the 4-phenylmidazole ligand and a good acceptor such as the tetrafluoroborate. In the case of nitrato and bis(acetato) derivatives the compounds are covalent with a 2:1, $3: 1$ or $4: 1$ ligand/metal ratio.

The compounds obtained are less soluble in common organic solvents and in water in conditions similar to the fisiologic ones and moreover they often presents hydrogen bond; these observations make 
imidazoles potential models for the study of biological systems, overall with acceptors such as $\mathrm{ZnCl}_{2}$ and $\mathrm{Zn}\left(\mathrm{CH}_{3} \mathrm{COO}\right)_{2}$.

\section{Acknowledgements}

We would like to thank the Italian Ministry of the University (MURST) and the University of Camerino for financial help.

\section{References}

1. C. F. Mills, ed., Zinc in Human Biology, Springer-Verlag, New York, 1989.

2. A. S. Prasad, Biochemistry of Zinc, Plenum, New York, 1993.

3. B. L. Vallee and D. S. Auld, Biochemistry, 1990, 29, 5647.

4. I. Bertini, H. B. Gray, S. J. Lippard, Bioinorganic Chemistry, University Science Books, Mill Valley, CA, 1994.

5. $\quad$ B. L. Vallee and D. S. Auld, Faraday Discuss., 1992, 93, 47.

6. B. L. Vallee and D. S. Auld, Proc. Natl Acad. Sci. USA, 1991, 88, 999

7. B. L. Vallee and W. Maret, in Metallothionem III, K. T. Suzuki, N. Imura and M. Kimura, eds., Bierkhäuser Verlag, Basel, Switzerland, 1993, 1-27.

8. B. L. Vallee, Neurochem. Int., 1995, 27, 23.

9. K. C. Mohapatra and K. C. Dash, J. Inorg. Nucl. Chem., 1977, 39, 1253.

10. P. Wardman, Int. J. Radiat. Biol., 1975, 28, 585.

11. P. B. Agscough. A. J. Elliot and G. A. Salman, Int. J. Radiat. Biol., 1975, 27, 603.

12. T. E. Creighton, Proteins, Structures and Molecular Properties, W. H. Freeman, New York, 1984.

13. W. Saenger, Principles of Nucleic Acid Structure, Springer-Verlag, New York, 1984.

14. J. M. Pratt, Inorqanic Chemistry of Vitamin $B_{12}$, Academic Press, New York, 1972.

15. There is a vast literature on this field, see for example: (a) W. R. Sheidt, S. R. Osvath and Y. J. Lee, J. Am. Chem. Soc., 1987, 109, 1958; (b) W. R. Sheidt, J. F. Kirner, J. L. Hoard and C. A. Reed, J. Am. Chem. Soc., 1987, 109, 1963; (c) M. Momenteau, W. R. Sheidt, C. W. Eigenbrot, and C. A. Reed, J. Am. Chem. Soc., 1988, 110, 1207, and references reported therein.

16. A. Cingolani, A. Lorenzotti, D. Leonesi and F. Bonati, Gazz. Chim. Ital., 1981, 111, 243.

17. A. Cingolani, A. Lorenzotti, D. Leonesi and F. Bonati, Inorg. Chim. Acta, 1984, 81, 127.

18. A. Cingolani, A. Lorenzotti, D. Leonesi and F. Bonati, Inorg. Chim. Acta, 1984, 89, 113.

19. D. Leonesi, A. Lorenzotti, A. Cingolani and F. Bonati, Gazz. Chim. Ital., 1981, 111, 483.

20. C. Pettinari, G. Gioia Lobbia, G. Sclavi, D. Leonesi, M. Colapietro and G. Portalone, Polyhedron, $1995,14,1709$.

21. C. Pettinari, F. Marchetti, R. Polimante, A. Cingolani, G. Portalone and M. Colapietro, Inorg. Chim. Acta, 1996, 249, 215.

22. C. Pettinari, R. Pettinari, M. Pellei and G. Gioia Lobbia, Polyhedron, 1999, 18, 1941.

23. A. Cingolani, Effendy, F. Marchetti, C. Pettinari, B. W. Skelton and A. H. White, J. Chem. Soc., Dalton Trans., 1999, 4047.

24. C. Pettinari, F. Marchetti, A. Cingolani and S. Bartolini, Polyhedron, 1996, 15, 1263.

25. C. Pettinari, F. Marchetti, M. Pellei, A. Cingolani, L. Barba and A. Cassetta, J. Orqanomet. Chem., 1996, 515, 119.

26. C. Pettinari, M. Pellei, F. Marchetti, C. Santini and M. Miliani, Polyhedron, 1998, 17, 561.

27. C. Pettinari, M. Pellei, M. Miliani, A. Cingolani, A. Cassetta, L. Barba, A. Pifferi and E. Rivarola, $J$. Orqanomet. Chem., 1998, 553, 345.

28. C. Pettinari, M. Pellei, C. Santini, I. Natali, F. Accorroni and A. Lorenzotti, Polyhedron, 1998, 17, 4487.

29. C. Pettinari, F. Marchetti, A. Cingolani, S. I. Troyanov and A. Drozdov, Pohyhedron, 1998, 17, 1677.

30. K. Shobatake, C. Postmus, J. R. Ferraro and K. Nakamoto, Appl. Spectroscopy, 1969, 23, 12.

31. J. Bradbury, K. P. Forest, R. H. Nuttal and S. W. A. Sharp, Spectrochim. Acta, 1967, 23, 2701.

32. B. C. Cornilsen and K. Nakamoto, J. Inorg. Nucl. Chem., 1974, 36, 2467.

33. K. Nakamoto, Infrared and Raman Spectra of Inorganic and Coordination Compounds, 4th edn., J. Wiley \& Sons, U.S.A., 1986.

34. M. R. Rosenthal, J. Chem. Ed., 1973, 50, 331.

35. C. C. Addison, N. Logan, S. C. Wallwork and C. D. Garner, Quart. Rev., 1971, 25, 289.

36. A. B. P. Lever, E. Mantovani and B. S. Ramaswamy, Can. J. Chem., 1971, 49, 1957.

37. A. N. Chebotarev, M. V. Shestakova, T. M. Shcherbakova, V. F. Khorunov and V. I. Nikitin, Russian J. Inorq. Chem., 1993, 38, 253.

38. A. A. G. Tomlinson, M. Bonamico, G. Dessy, V. Fares, and L. Scaramuzza, J. Chem. Soc., Dalton Trans., 1972, 1671.

39. G. B. Deacon and R. J., Phillips, Coord. Chem. Rev., 1980, 33, 227.

40. C. Pettinari, A. Lorenzotti, M. Pellei and C. Santini, Polyhedron, 1997, 16, 3435 and references therein.

41. N. F. Borkett and M. 1. Bruce, J. Orqanomet. Chem., 1974, 65, C51.

42. R. Gassend, J. C. Marie and J. C. Pommier, J. Orqanomet. Chem., 1977, 132, 69. 
43. M. A. Angaroni, G. A. Ardizzoia, T. Beringhelli, G. D'Alfonso, G. La Monica, N. Masciocchi and M. Moret, J. Orqanomet. Chem., 1989, 363, 409.

Received: September 1, 2000 - Accepted: September 12, 2000 Accepted in publishable format: September 20, 2000 